\title{
Short reads from honey bee (Apis sp.) sequencing projects reflect microbial associate diversity
}

\author{
Michael Gerth Corresp., ${ }^{1}$, Gregory DD Hurst ${ }^{1}$ \\ ${ }^{1}$ Institute of Integrative Biology, University of Liverpool, Liverpool, United Kingdom \\ Corresponding Author: Michael Gerth \\ Email address: gerth@liverpool.ac.uk
}

High throughput (or 'next generation') sequencing has transformed most areas of biological research and is now a standard method that underpins empirical study of organismal biology, and (through comparison of genomes), reveals patterns of evolution. For projects focused on animals, these sequencing methods do not discriminate between the primary target of sequencing (the animal genome) and 'contaminating' material, such as associated microbes. A common first step is to filter out these contaminants to allow better assembly of the animal genome or transcriptome. Here, we aimed to assess if these 'contaminations' provide information with regard to biologically important microorganisms associated with the individual. To achieve this, we examined whether the short read data from Apis retrieved elements of its well established microbiome. To this end, we screened almost 1000 short read libraries of honey bee (Apis sp.) DNA sequencing project for the presence of microbial sequences, and find sequences from known honey bee microbial associates in at least $11 \%$ of them. Further to this, we screened $\sim 500$ Apis RNA sequencing libraries for evidence of viral infections, which were found to be present in about half of them. We then used the data to reconstruct draft genomes of three Apis associated bacteria, as well as several viral strains de novo. We conclude that 'contamination' in short read sequencing libraries can provide useful genomic information on microbial taxa known to be associated with the target organisms, and may even lead to the discovery of novel associations. Finally, we demonstrate that RNAseq samples from experiments commonly carry uneven viral loads across libraries. We note variation in viral presence and load may be a confounding feature of differential gene expression analyses, and as such it should be incorporated as a random factor in analyses. 
1 Short reads from honey bee (Apis sp.) sequencing projects reflect microbial associate

2 diversity

3

4 Michael Gerth* \& Gregory D. D. Hurst

5

6 University of Liverpool, Institute of Integrative Biology, Biosciences Building, Crown Street

7 Liverpool L69 7ZB, United Kingdom

8

9

10

11

12

13

$14 *$ Correspondence \& material requests

15 gerth@liv.ac.uk 


\section{Abstract}

17 High throughput (or 'next generation') sequencing has transformed most areas of biological

18 research and is now a standard method that underpins empirical study of organismal biology, and

19 (through comparison of genomes), reveals patterns of evolution. For projects focused on animals,

20 these sequencing methods do not discriminate between the primary target of sequencing (the

21 animal genome) and 'contaminating' material, such as associated microbes. A common first step

22 is to filter out these contaminants to allow better assembly of the animal genome or

23 transcriptome. Here, we aimed to assess if these 'contaminations' provide information with

24 regard to biologically important microorganisms associated with the individual. To achieve this,

25 we examined whether the short read data from Apis retrieved elements of its well established

26 microbiome. To this end, we screened almost 1000 short read libraries of honey bee (Apis sp.)

27 DNA sequencing project for the presence of microbial sequences, and find sequences from

28 known honey bee microbial associates in at least $11 \%$ of them. Further to this, we screened $\sim 500$

29 Apis RNA sequencing libraries for evidence of viral infections, which were found to be present

30 in about half of them. We then used the data to reconstruct draft genomes of three Apis

31 associated bacteria, as well as several viral strains de novo. We conclude that 'contamination' in

32 short read sequencing libraries can provide useful genomic information on microbial taxa known

33 to be associated with the target organisms, and may even lead to the discovery of novel

34 associations. Finally, we demonstrate that RNAseq samples from experiments commonly carry

35 uneven viral loads across libraries. We note variation in viral presence and load may be a

36 confounding feature of differential gene expression analyses, and as such it should be

37 incorporated as a random factor in analyses. 


\section{Introduction}

Novel DNA sequencing methods have revolutionized biological and medical research in the last two decades (Goodwin et al. 2016). High throughput sequencing (or 'massively parallelized sequencing', 'next generation sequencing', 'NGS') facilitated the creation of enormous amounts of data for a fraction of the costs associated with traditional Sanger methods (Kircher \& Kelso 2010; Sboner et al. 2011). This 'genomics revolution', has not only enhanced our understanding of molecular and genome evolution (Wolfe \& Li 2003), but also contributed to the recognition that eukaryotes are commonly associated with a plethora of microbial taxa. (For the sake of simplicity, we will in this paper refer to all bacteria, microbial eukaryotes, and viruses as "microbes".)

In eukaryote genome sequencing projects, sequences deriving from these microbes may obstruct genome or transcriptome assembly efforts, and measures directed at removing microbial associates are routinely performed. This is achieved either by antibiotic treatment of the target organism prior to sequencing (Colbourne et al. 2011), or by removing microbial sequences bioinformatically after sequencing (Schmieder \& Edwards 2011). While eliminating microbes may facilitate eukaryotic genome reconstruction, it neglects the recently emerging appreciation of microbes as a biologically important component of all multicellular life. Numerous examples illustrate the impact of microbes on animal and plant biology, including physiology, behavior, and evolution (McFall-Ngai et al. 2013). These findings have led to a concept that defines an individual eukaryote with all its associated microbes (microbiome) as an entity (holobionthologenome) (Bordenstein \& Theis 2015). Although this concept is contentious (Moran \& Sloan 2015; Douglas \& Werren 2016), it is undisputed that some aspects of organismal biology can only be understood by deciphering interactions with microbial symbionts. 
61 To characterize microbiome composition, three approaches are commonly used. First,

62 microbes may be isolated from the host and cultured axenically. Their properties can then be

63 determined either through traditional microbiological methods or by sequencing (Browne et al.

64 2016). This approach has the benefit of providing both biological and genomic information, but

65 limits discovery to culturable taxa. Second, microbiome taxa may be identified by amplicon

66 sequencing. Specific primers are used to amplify a short informative region from all bacterial

67 taxa in a sample (usually a part of the 16S rRNA gene), and then sequenced (today typically via

68 NGS methods) (Caporaso et al. 2012). This mechanism discovers broad patterns of community

69 diversity, but at a coarse scale, and with weaker functional information. Finally, microbiome

70 composition can be determined via metagenomics, i.e., collective genome sequencing of all

71 bacteria present in a sample (Riesenfeld et al. 2004). This is unbiased, fine scaled, and provides

72 an assessment of biological potential at a community scale. Approaches to characterize viral

73 sequences found in the environment or hosts are similar to the ones used to detect bacterial

74 microbes described above and metagenomics is now most commonly employed for this task

75 (Delwart 2007; Mokili et al. 2012).

76 In this study, we examined if the data generated in eukaryotic sequencing projects can be

77 used to identify microbiome taxa and harness genomic data. Previously, this approach was used

78 to recover genomes of heritable microbes that occur in high densities in many arthropod species,

79 and are therefore prone to be retrieved in arthropod sequencing projects. For example, the

80 genomes of multiple Wolbachia strains were discovered in Drosophila sequencing data,

81 revealing novel Wolbachia diversity and patterns of Wolbachia evolution (Salzberg et al. 2005;

82 Richardson et al. 2012; Siozios et al. 2013). Furthermore, the software packages such as

83 Glimmer (Delcher et al. 2007) and Blobtools/Blobology (Kumar et al. 2013) were specifically 
84 designed to facilitate and automated the extraction of symbiont genomes from NGS data, and

85 subsequent studies have demonstrated their efficiency in this task (Blow et al. 2016; Denver et

86 al. 2016; Fierst et al. 2017). In bee sequencing projects, (partial) symbiont genomes were

87 previously detected in bumble bees (Martinson et al. 2014), a mason bee (Gerth et al. 2014), and

88 the Apis mellifera genome (Cox-Foster et al. 2007).

89 Here, we systematically examine a large number of short reads of honey bee (Apis sp.)

90 sequencing projects to investigate whether this archived data can be used to retrieve a wider set

91 of microbial associates, including pathogens and gut symbionts. We focus on honey bees

92 because 1) there is a large number of short read sequencing projects targeting Apis; 2) the

93 components of healthy and unhealthy Apis microbiomes are well established (Evans \& Schwarz

94 2011; Kwong \& Moran 2016); 3) managed populations of the economically important honey

95 bees have been in decline worldwide (Neumann \& Carreck 2010), and it was hypothesized that

96 certain bacteria and viruses are key players in this decline (Cox-Foster et al. 2007). Thus, any

97 novel genomic data on honey bee symbionts may directly contribute to our understanding of bee

98 disease.

99 To identify 'contaminants', we here use 'bait' sequences of symbionts and pathogens to

100 screen a large number of short read libraries from Apis sequencing projects. We demonstrate that

101 the libraries contain non-target sequences from many sources, some of which reflect the natural

102 honey bee microbiome and virome. We further show that highly covered, and possibly novel

103 symbiont genomes can be retrieved from this contamination. Our study highlights the value of

104 database sequences for exploratory symbiont screens and argues against neglecting the filtered

105 'contaminants' in sequencing projects. 
106 Materials \& methods

107

108 Screening for microbes in Apis short read sequencing libraries

109 A graphical overview of our screening process can be found in Fig. 1. We compiled two

110 sets of reference sequences to be used as databases for subsequent screens (Fig. 1). First, for 15

111 common Apis associated symbionts and pathogens, we used one short signature sequence each

112 instead of complete genomes, in order to reduce the computational expense of all following

113 steps. These were of slowly evolved ribosomal RNA genes to allow a range of diversity to be

114 recovered through sequence similarity to the bait. Second, for 11 common viral associates, we

115 compiled complete genomes. As all of these were very small ( $10,000 \mathrm{bp})$, this was unlikely to

116 significantly impact computational run times. Fasta files of compiled database sequences are

117 available under https://github.com/gerthmicha/symbiont-sra.

118 Next, we searched for honey bee sequencing projects in NCBI's short read archive, using

119 the search term 'Apis', and created lists of the hits for DNA and RNA sequencing projects, which

120 were subsequently processed separately (Fig. 1). The great majority of projects were from Apis

121 mellifera samples, but a few RNA sequencing projects from Apis cerana and Apis florea were

122 also included. We will from here on refer to all of those samples as "Apis". From the list of DNA

123 sequencing projects, we excluded metagenome and amplicon projects, as these are specifically

124 aimed at microbiome taxa. At the time of the search (October 2015), 18 projects matched these

125 criteria. We downloaded all short read libraries associated with these projects (993 in total, Table

126 S1) and mapped all reads of each of the libraries to the 'symbionts \& pathogens' reference

127 database using NextGenMap version 0.4.12 (Sedlazeck et al. 2013). If at least 1,000 reads of a

128 library were aligned to one or more sequence baits, we extracted the matching reads and

129 assembled them using SPAdes version 3.7 (Bankevich et al. 2012). Contigs resulting from this 
130 assembly were then subject to taxonomic annotation via BLAST+ (Camacho et al. 2009)

131 searches against a local copy of the NCBI 'nt' database, and the Blobtools package (Kumar et al.

132 2013).

133 For the RNA dataset, we removed any library from projects investigating the effects of

134 viruses or Varroa infections, as Varroa treatment usually results in viral infections (Allen \& Ball

135 1996). Next, the reads of all RNA sequencing libraries (492 libraries from 58 projects in total,

136 Table S2) were mapped against the viral database as described above, and the number of mapped

137 reads was counted using samtools version 1.4 ( $\mathrm{Li}$ et al. 2009). Mapped reads were then

138 assembled using IDBA-UD (Peng et al. 2012) and MEGAHIT version 1.1.1 (Li et al. 2015),

139 which were designed to handle highly uneven sequencing depths, as expected from viral data

140 (Visser et al. 2016). The 'better' assembly from these two methods was chosen by means of viral

141 contig length comparison, i.e., the assembly that created the single longest contig was kept. This

142 was more appropriate than choosing the highest N50 value, as most libraries contained only a

143 single viral associate. If the two assembly methods produced overlapping contigs, which were

144 identical in the overlap, the contigs were merged. A detailed description of all steps used to

145 screen for microbial associates can be found under https://github.com/gerthmicha/symbiont-sra.

147 Assessing Lactobacillus diversity in Apis DNA sequencing libraries

148 Since our initial screen of DNA sequencing libraries yielded a high number of hits to

149 various Lactobacillus species, we repeated the entire procedure using a database of $62016 \mathrm{~S}$ bait

150 sequences from Lactobacillus only. These sequences were taken from a previously compiled

151 dataset of Lactobacilli associated with Apis, other Hymenoptera, and other Lactobacillus

152 sequences retrieved from public databases (McFrederick et al. 2013). All hits shorter than 250bp 
153 were discarded, and remaining contigs were combined with the reference sequences. We used

154 SSU-ALIGN version 0.1 (Nawrocki 2009) to align and mask this dataset based on conserved

155 secondary structure. Original and masked alignments are available from

156 https://github.com/gerthmicha/symbiont-sra. A maximum likelihood phylogeny was

157 reconstructed from the complete $16 \mathrm{~S}$ alignment (740 sequences in total) using IQ-TREE version

158 1.3.10 (Nguyen et al. 2015) with automated model selection and 1,000 ultrafast bootstraps (Minh

159 et al. 2013) to assess node support. The resulting tree was visualized using the online tool

160 Evolview (He et al. 2016). Furthermore, as an approximate measure for the number of

161 Lactobacillus OTUs recovered with our approach, we used the average neighbor clustering

162 algorithm as implemented in mothur version 1.34.4 (Schloss et al. 2009).

163 Although our aim was not to recover all, but only the highly covered symbiont data from

164 honey bee short reads, we wanted to test if our screening approach yields comparable results to

165 more commonly used metagenomic approaches. To this end, we screened the reads of a

166 metagenomic dataset created from the pooled DNA of 150 honeybee worker hindguts (Engel et

167 al. 2012; 43M 150bp paired-end reads, SRA accession: SRR5237156) for Lactobacillus in the

168 same way as described above. We found 6 different Lactobacillus $16 \mathrm{~S}$ sequences, all within the

169 Firm-4 and Firm-5 Lactobacillus groups (Fig. S1). This was in agreement to the results obtained

170 from taxonomic profiling approaches performed by Engel et al. (2012) and thus confirmed the

171 general effectiveness of our approach (Fig. S1).

172

173 Reconstruction of symbiont genomes from Apis DNA sequencing libraries

174 Next, we aimed to validate that whole symbiont genomes can in principle be recovered

175 from Apis sequencing projects. To this end, we chose one Apis mellifera intermissa sequencing 
176 library (SRR1046114, 85.5M 100bp paired-end reads) that contained 'contamination' from two

177 Lactobacillus strains (Lactobacillus kunkeei \& Fructobacillus sp.). We performed a de novo

178 assembly using all reads with MEGAHIT version 1.1.1 (Li et al. 2015). All resulting contigs of

179 this assembly were taxonomically assigned to either L. kunkeei, Fructobacillus sp. or 'other'

180 based on BLAST searches, GC distributions, and read coverage. Reads matching to contigs from

181 either Lactobacillus strain were then separately re-assembled using SPAdes, and all contigs

182 smaller than 500bp discarded. Completeness and contamination of the novel draft genomes were

183 assessed based on the presence of conserved marker genes using CheckM version 1.0.6 (Parks et

184 al. 2015), and annotation performed with PROKKA version 1.12 (Seemann 2014). The annotated

185 draft genomes are available under under https://github.com/gerthmicha/symbiont-sra. To

186 evaluate the evolutionary relationships of newly assembled genomes in a broader taxonomic

187 context, we assessed their phylogenetic placement. Whole-genome datasets were compiled for

188 both strains (13 L. kunkeei genomes, 9 Fructobacillus \& Leuconostoc genomes altogether, Table

189 S3). For each of the datasets, single copy orthologs were identified using OrthoFinder version

$190 \quad 0.2 .8$ (Emms \& Kelly 2015). Recombining loci were identified by using the pairwise homoplasy

191 index test (Bruen et al. 2006), and removed from subsequent analyses (window size $=20$ amino

192 acid positions, significance cutoff at 0.05). Using IQ-TREE, we performed maximum likelihood

193 analysis of two final supermatrices (947 loci and 290,774 aa for the L. kunkeii dataset, 435 loci

194 and 145,069 positions for the Fructobacillus/Leuconostoc dataset). Prior to this, best-fitting

195 partitioning schemes and models were selected using the 'greedy' scheme implemented in IQ-

196 TREE (Lanfear et al. 2012).

197 Using the same approach, we assembled and annotated a Spiroplasma melliferum genome

198 from the Apis mellifera library SRR957082, ( 224.5M 50bp single end reads). Phylogenetic 
199 analysis was performed based on a dataset of 206 concatenated single copy genes $(58,950$ amino

200 acid positions) shared among 17 Spiroplasma strains (Table S3). Furthermore, to assess synteny,

201 the newly assembled draft genome was ordered against and aligned with other Spiroplasma

202 melliferum genomes (one genome each of strains IPMB4A and KC3) using the

203 progressiveMauve algorithm of Mauve development snapshot version 2015-02-13 (Darling et al.

204 2010).

205

206

Assessing viral diversity in Apis RNA sequencing libraries

207 All potential viral contigs resulting from the assembly steps outlined above were blasted against a local copy of NCBI's 'nt' database. As most contigs were annotated as deformed wing virus (DWV), we reconstructed the phylogenetic relationships between the detected DWV strains. We used only contigs where at least half of the DWV genome was present $(5,000 \mathrm{bp}$

211 minimum length), and aligned all sequences using MAFFT. As a reference, we added complete

212 genomic sequences for each of the three types of DWV (A, B or VDV-1- Varroa destructor virus

213 1, and C) and one for Kakugo virus (KV), which is a variant of DWV type A (Mordecai et al.

214 2016). The phylogeny was estimated via maximum likelihood with IQ-TREE as described 215 above.

217 Exploring viral effects on Apis gene expression from RNA sequencing libraries

218 We observed that in some cases, libraries from a single project (i.e., NCBI's BioProject)

219 displayed pronounced differences in viral loads as measured in our screen. We thus hypothesized

220 that these differences should also be detectable through differentially expressed host genes. To

221 test this hypothesis, four projects were investigated (Table 1) in which viral loads were 
222 particularly uneven (proportion of viral reads across libraries $0 \%-37 \%, 0 \%-15 \%, 0 \%-53 \%$, and

$2230 \%-32 \%$, respectively- see Table S4 for details). Several studies have examined differential

224 gene expression of honey bees in response to viral infection, and a set of genes commonly

225 responding to pathogens was recently identified via meta-analyses (Doublet et al. 2017). We

226 used the 20 genes that were consistently differentially expressed across multiple studies (first 20

227 ranks in the category 'differentially-regulated' from the Doublet et al. 2017 analysis). The

228 relative expression levels of these genes were determined for all libraries of the selected projects

229 by 1) quality trimming of all reads with cutadapt version 1.1.3 (Martin 2011; options: -q 25,25 --

230 minimum-length 50 --pair-filter=both) 2) mapping the reads with NextGenMap (min. identity

$23199 \%$ ); 3) counting the reads with samtools; 4) normalizing read counts to reads per kilobase per

232 million mapped reads (RPKM) and $\log 2$ transforming this value, 5) allocating the libraries from

233 each project to either 'virus' or 'virus-free' groups based on the proportion of viral reads in the

234 libraries; 6) testing for differentially expressed genes between these groups using the qCML

235 method and exact tests in the R package 'edgeR'(Robinson \& Smyth 2007; Robinson et al. 2009;

236 R Core Team 2015). Genes were regarded as differentially expressed for P-values $\leq 0.05$.

\section{Results}

238 Using bait sequences of 15 common Apis- associated microbes, we found non-target

239 symbiont sequence data in 105 of the 993 investigated DNA sequencing libraries $(\sim 11 \%)$.

240 Assembly of symbiont reads resulted in a total of 782 contigs from these 105 libraries (1-57

241 contigs per library, Table S5), which were on average 371 bp long (34-1670 bp, Table S5).

242 Taxonomic annotation revealed that these contigs originated from either Apis- associated taxa

243 that were targeted with our bait sequences, or from other sources for which there is no current 
244 evidence of Apis association (Fig. 2). Of the former, we detected most common gut symbionts

245 known to be associated with honey bees (Kwong \& Moran 2016), i.e., Bartonella,

246 Bifidobacterium, Frischella, Gilliamella, Lactobacillus, and Snodgrassella, but not

247 Parasaccharibacter. Of the pathogens screened for, we detected Crithidia/Lotmaria (which are

248 inconsistently annotated in NCBI's nucleotide database and therefore not differentiated here;

249 Schwarz et al. 2015), Nosema, and Spiroplasma (Fig. 2). We did not find evidence for

250 chalkbrood, American foulbrood, or European foulbrood in the investigate DNA libraries

251 (Ascosphaera, Paenibacillus, and Melissococcus, respectively). Sequences from organisms not

252 naturally associated with honey bees included those from fungi (Ascomycota) and plants, that

253 were likely not part of the native microbiome of the sequenced samples. These contaminations

254 were crossed-checked via manual online BLAST searches and were confirmed to represent 'true'

255 hits with high and continuous identities with the respective database sequences.

256 Because the majority of hits in the DNA sequencing libraries were Lactobacilli, we repeated

257 the screening, this time using only Lactobacillus $16 \mathrm{~S}$ sequences as baits. We found 121

258 Lactobacillus sequences in 40 of the 993 investigated libraries, corresponding to 25 OTUs

259 (estimated with mothur using a 5\% cutoff). In our phylogenetic analysis based on 16S rRNA

260 sequences, most of the detected strains clustered within Lactobacillus groups known to be

261 associated with honey bees (Fig. 3a). Of the recovered sequences not clustering within these

262 lineages, three were found to group with other Apis- associated Lactobacilli as sister group to the

263 Lactobacillus coryniformis group (Fig. 3a). Online BLAST searches revealed Fructobacillus

264 species as closest matches based on 16S rRNA sequence.

265 Next, we aimed at recovering draft genome sequences of bee-associated Lactobacilli. We

266 chose an Apis mellifera intermissa sequencing library from which $16 \mathrm{~S}$ sequences of both $L$. 
267 kunkeei and Fructobacillus isolates were detected in our screen. The contigs of a meta-assembly

268 were taxonomically annotated, and reads matching to the respective target taxa were then

269 assembled and annotated separately. For each assembly, we performed a phylogenetic analysis

270 based on all single copy orthologs shared with related genomes (Fig. 3b, c), thus confirming the

271 identity of the strains as L. kunkeei (Fig. 3b) and Fructobacillus (Fig. 3c). Both genomes were

272 highly covered and mostly complete based on the presence of conserved markers (Fig. 3d).

273 Finally, we recovered the genome of a Spiroplasma melliferum strain from another Apis

274 sequencing library (Fig. 4). In the meta-assembly, Spiroplasma and Apis contigs could be clearly

275 separated by coverage and taxonomic annotations (Fig. 4b). The refined assembly resulted in a

276 highly covered draft genome of Spiroplasma melliferum, which is very similar to the two

277 previously sequenced Spiroplasma melliferum strains (Alexeev et al. 2012; Lo et al. 2013), based

278 on shared ortholog clusters, genome organisation, and phylogeny (Fig. 4a, c, d).

279 The second part of our screen was focused on viruses in honey bee RNA sequencing

280 libraries. Here, whole viral genomes were used as baits, and viral reads were found in about half

281 of the investigated libraries (236/492 libraries with 500 or more viral reads). Deformed wing

282 virus type A (DWV A) was by far the most frequently found virus, and was present in almost all

283 libraries that contained viral reads. We found evidence for all other tested honey bee viruses as

284 well, albeit at a lower frequency (Fig. 5a). In many cases, the viral reads made up only a

285 negligible proportion of a sequencing library (Fig. 5b). However, in 75 RNA libraries, more than

$2865 \%$, and up to $54 \%$ of the reads were of viral origin, indicating substantial viral loads in the

287 honey bees from which the samples were originally prepared (Fig. 5b). We next investigated the

288 genetic diversity of DWV variants by assembling draft genomes from the viral reads extracted

289 from RNA sequencing libraries. Our phylogenetic analysis showed a clear separation between A 
290 and B types of DWV and considerable genetic variation is evident especially in the DWV A

291 samples (Fig. 6). Furthermore, viruses extracted from libraries of the same project were often

292 phylogenetically closely related (Fig. 6), which is not surprising given that samples in one

293 project were commonly from a single geographic location (Table S2).

294 Viral infections usually result in a host response that should be measurable by means of

295 differentially expressed genes (e.g., immunity-related genes). We tested this prediction using

296 RNA sequencing libraries from four experiments (NCBI's BioProjects) that showed strong

297 variations in viral loads across libraries, and 20 candidate loci that were previously found to be

298 differentially expressed in response to pathogens (Doublet et al. 2017; see Table S6). When

299 comparing RNA sequencing libraries with and without viruses, 15 of these genes were

300 differentially expressed in at least one of the investigated experiments (Table 2), and one gene

301 was found to be differentially expressed in all of them (Table 1). This gene (LOC410087) is not

302 functionally characterized in honey bees, but likely encodes a protein related to heat shock

303 response, which may be activated during viral infections (Merkling et al. 2015). Within each

304 experiment, at least five of the investigated genes were differentially expressed between the two

305 groups (Table 1). Relative expression rates of three exemplary genes that were significantly

306 different between libraries with and without viral reads are plotted for one project in Fig. 7.

307 Expression plots for all genes from all investigated projects are shown in Figs. S2-S5.

308 Discussion

309 Apis DNA sequencing libraries can help to reconstruct the honey bee microbiome

310 We used bait sequences of Apis symbionts and pathogens to determine if microbial data can

311 be retrieved from DNA sequencing projects targeting Apis (honey bees) and found evidence for 
312 the presence of these taxa in $11 \%$ of 993 Apis short read libraries. This measure of non-target

313 'contamination' can be considered as conservative, since our approach only reports relatively

314 high levels of contamination (at least 1000 reads per bait sequence). Our approach revealed that

315 all common gut symbionts of honey bees are also present as 'contamination' in DNA sequencing

316 libraries (Fig. 2). Furthermore, the relative frequency of each of the gut colonizers in focused

317 study roughly corresponds to the frequencies at which we detected them. For example, the

318 microbiome of healthy honey bees is dominated by Lactobacilli (Kwong \& Moran 2016), and

319 this is also reflected in our results (Fig 2, Table S5). Our findings demonstrate that a reasonable

320 understanding of honey bee gut microbiome composition could be gained solely from non-target

321 sequences produced as a by-product of honey bee sequencing projects.

322 When we next targeted our screen of DNA sequencing libraries only at Lactobacillus, our

323 protocol detected 25 taxonomically different Lactobacillus strains. Phylogenetic reconstruction

324 of Lactobacillus relationships based on 16S rRNA generally reflected the current understanding

325 of this genus' taxonomy (Felis \& Dellaglio 2007; Salvetti et al. 2012), and revealed that most

326 Lactobacilli known to be associated with honey bees are also present in Apis short read libraries.

327 This includes Firm-4 and Firm-5 Lactobacilli, both of which are honey bee hindgut colonizers,

328 and L. kunkeei, which is common in nectar and hive material, and sometimes found in honey bee

329 crops (Kwong \& Moran 2016). Furthermore, we found Fructobacillus, which share an ecological

330 niche with L. kunkeei, i.e., they are found in flowers, nectar, and in honey bee guts (Endo et al.

331 2009; Endo \& Salminen 2013). Although not classified as such, recent phylogenomic evidence

332 suggests that Fructobacillus (and the closely related Leuconostoc) are part of the Lactobacillus

333 radiation (Sun et al. 2015). Here, we also infer Fructobacillus grouping within, rather than

334 outside of Lactobacillus (Fig. 2a). 
In addition to the gut symbionts, three common honey bee pathogens were detected with

336

337

338

339

340

341

342

343

344

345

346

347

348

349

350

351

352

353

354

355

356 357

our approach: Nosema, Crithidia/Lotmaria, and Spiroplasma. Nosema are microsporidian gut

parasites of various honey bee species, and while the sampling of our screen is not

representative, this finding corroborates the recognition of Nosema as widespread pathogen of honey bee colonies worldwide (Nixon 1982; Klee et al. 2007). Crithidia/Lotmaria

(Trypanosomatidae), another gut pathogen of Apis and related bee species (Schwarz et al. 2015) was detected at an even higher frequency (Fig. 1, Table S5). We further found Spiroplasma melliferum in one of the investigated sequencing libraries. Spiroplasma are common symbiotic bacteria of many invertebrates (Duron et al. 2008) and have been connected to pathogenicity in honey bees (Clark 1977). The bait sequences of all of these pathogens showed a high coverage in our screen, suggesting that novel genetic variants can be recovered from already available data, or from data that will become available as by-product of future honey bee sequencing projects. In addition to the targeted microbes, a number of taxa that are likely not part of the natural Apis microbiome were detected. For example, we detected Aspergillus in several sequencing libraries that originated from museum material, which likely represents post mortem saprophytic growth. We also retrieved hits to plant sequences which might originate from co-amplified and sequenced pollen DNA (Fig. 2). This 'false discovery' illustrates an important caveat in our approach: the differentiation between host-associated microbes and microbes from other sources may not always be possible, and will be particularly difficult for museum specimens. Though not problematic in the examples we present, the situation is likely more complicated for study of host species with a less well-investigated microbiome, or for symbionts that are very similar to environmental taxa. In these cases, the approach will establish candidates that will then require direct validation. 
Finally, as demonstrated previously in other taxa with very similar approaches (Kumar et al.

359

360

361

362

363

364

2013), we show that draft genomes of microbial symbionts can be recovered from Apis short

reads. For example, inspecting the non-target components of just a single Apis mellifera

intermissa sequencing library produced novel, highly covered, and near complete draft genomes

of Lactobacillus kunkeii and a Fructobacillus strain (Fig. 3 b,c,d). Although the 16S sequence of

the Fructobacillus strain best matched $F$. fructosus, our analysis suggests it belongs to a species

so far not represented by genomic sequences in public databases, or even a novel species (Fig.

3c). Conceivably, many additional Lactobacillus variants could be retrieved from the libraries

investigated here, potentially providing a more complete picture of the Apis microbiome

composition and function. It should be noted that draft genomes reconstructed this way must be

regarded as 'population consensus' genomes, as opposed to genomes sequenced from cultured

bacterial clones. While these genomes cannot be linked to a bacterial clone, they still provide information of metabolic capacities within the Apis microbiome.

In summary, our analyses suggest that 'contamination' from honey bee sequencing projects

can help to characterize the honey bee microbiome, and inform about its composition, abundance of specific taxa, and through recovery of genomes, about metabolic capacities. Short read repositories thus provide plenty of biological information on known associates, but also on undescribed ones. If, for example, a novel microbial associate of honey bees is discovered, one could potentially use the available short read data (and associated metadata) to learn about its distribution, prevalence, and genome. Or, alternatively, one could confirm the absence of certain taxa with more confidence. Kwong et al. (2017) identified Bombiscardovia sp. and Schmidhempelia sp. as gut microbes of bumble bees (Bombus sp.) that are typically not present 
380 honey bees. We also did not find evidence for these taxa in Apis with our screening approach and

381 thus provide further support for their absence based on a large sample size.

382

383 Implications of viral reads as common contaminations in Apis RNA sequencing libraries

384 We found viral reads in about half of the 498 investigated RNA libraries, and DWV type A

385 as the most common viral associate, followed by DWV type B (=Varroa destructor virus-1), and

386 all other viruses we screened for (Fig. 5). DWV has a global distribution (Genersch \& Aubert

387 2010) and its transmission is facilitated by Varroa mites, which are also globally distributed

388 (Martin et al. 2012; Wilfert et al. 2016). Our findings are thus in line with these previous

389 observations. The detection of DWV despite our deliberate exclusion of RNA sequencing

390 experiments which investigated Varroa or virus treatments can be explained by the fact that, in

391 many cases, DWV infections are asymptomatic (Lanzi et al. 2006). The low proportion of DWV

392 reads found in many samples (as a proxy for viral load) also argues for asymptomatic infections

393 in the bees from which the RNA was extracted (Fig. 5). However, we found a number of RNA

394 sequencing libraries in which DWV read numbers made up a very large proportion of the reads

395 (10-54\%, Fig. 5). Very high viral titers are typically found in symptomatic bees (Chen et al.

396 2005; Lanzi et al. 2006; Brettell et al. 2017), and inspection of the metadata associated with

397 RNA libraries showing high proportions of viral reads revealed that many of these were created

398 from heads or brains (out of 58 libraries with $>10 \%$ viral reads, 25 from heads, 10 from other

399 tissues, 23 undisclosed, Table S4). This is notable because high DWV titres in honey bee heads

400 have been found to correlate with deformed wing phenotypes (Yue 2005; Zioni et al. 2011). This

401 suggests that for these specific experiments, RNA may have been extracted from DWV infected

402 bees despite obvious symptoms (i.e, crippled wings). 
Varroa mites and DWV are considered one of the main factors driving the decline in

404 managed honey bee populations (VanEngelsdorp et al. 2009; Conte et al. 2010), and molecular

405 data have been used to trace the global spread of these pathogens (Martin et al. 2012; Wilfert et

406 al. 2016). We here showed that almost complete DWV draft genomes can be extracted from

407 short read data, revealing considerable genetic diversity between the strains (Fig. 6). Archived

408 RNA sequencing reads of honey bees are thus a valuable, largely untapped resource for genomic

409 data of viruses. As described above for symbionts in DNA sequencing libraries, these data

410 inform about host association, distribution, and genetic diversity of viruses.

411 Furthermore, the detection of large amounts of viral reads in honey bee RNA sequencing

412 libraries has other, more direct implications. Differential gene expression analysis was

413 previously used to study e.g., development, learning, caste differences, and other behavioral

414 traits in honey bees (Whitfield 2003; Liu et al. 2011; Liang et al. 2012; Cameron et al. 2013;

415 Naeger \& Robinson 2016). It has further been established that viral infections lead to

416 transcriptomic responses in honey bees, some of which are conserved and universal for all

417 pathogens, while others are specific with respect to the pathogen present (summarized in Doublet

418 et al. 2017). Following from this, the presence of viruses alters transcriptomic profiles of honey

419 bees and therefore encorporated into analyses of differential gene expression, e.g., by adding

420 viral load as one factor in the interpretation. In most experiments analysed here, the presence of

421 viruses was likely not a problem, as viral reads were uniformly low across all samples. However,

422 we did identify some examples in which uneven viral loads very likely had an effect on

423 transcriptomic profiles (Tables $1 \& 2$, Figs. $7 \&$ S2-5). In these examples, the presence of

424 viruses, if unaccounted for, potentially leads to erroneous interpretations of the RNAseq data. 
425 Consequently, viruses should be screened for and identified in any RNA sequencing experiment

426 investigating differential gene expression.

427

\section{Conclusion}

The biological properties of an individual are a composite of the functions encoded in their genome and that of microbial associates, both symbionts and pathogens. We here revisited published short read data from Apis spp. sequencing projects to investigate if these give insight into the wider set of associates that are commonly disgarded as 'contaminants'. We found that a large variety of distinct Apis-associated microbial symbionts and pathogens can be detected as 'contamination' in these data. Further, due to the large depths of today's sequencing projects, the genomes of some microbial associates (which are typically much smaller than the target genomes) can often be recovered in high quality. Honey bees have a comparatively simple microbiota (Kwong \& Moran 2016) and are thus considered suitable models for microbiomeanimal interactions and evolution (Engel et al. 2016). Their enormous economic importance

439 (Calderone 2012), has driven the large number of honey bee sequencing projects. Our examination of the output of these projects suggests that substantial amounts of genomic information on bee-associated microbes are included in these data. While genomes gained from contaminated bee samples cannot and should not replace focused microbiological and metagenomic investigations, they might still improve our understanding of honey bee 444 microbiome composition and functioning.

445 Although our study was focused on Apis, it is conceivable that the amount of non-target

446 'contamination' is similar for other sequencing projects. As a best practice, and potentially 
447 rewarding research avenue in any sequencing project, we therefore suggest that all non-target

448 taxa should be identified, and their genomes assembled, annotated, and published alongside the

449 target genome. This requires less effort than it may seem, as de-contamination is already a

450 standard post-processing step. Instead of discarding the contaminated reads, they can be

451 processed with one of many available software solutions that automate the process of identifying

452 and assembling genomes from metagenomes (Oulas et al. 2015), thus minimizing the additional

453 workload. Not only would this provide the community with valuable genomic data of symbionts

454 from known host taxa, but it can additionally be argued that this is the most sensible thing to do

455 from a biological point of view. Evidence is mounting that symbiotic microbes influence almost

456 all aspects of their host's biology (Douglas 2014; Bordenstein \& Theis 2015). Taking into

457 account the total genomic information recovered in sequencing projects may therefore provide a 458 more complete picture of the target organism's biology.

\section{Acknowledgements}

460 The authors would like to thank Dr Seth Barribeau for comments on an earlier version of this

461 manuscript, and anonymous reviewers for constructive feedback. We further thank Dr Philipp

462 Engel and Olivier Emery for providing access to short read data.

463

\section{References}

465 Alexeev D, Kostrjukova E, Aliper A, Popenko A, Bazaleev N, Tyakht A, Selezneva O, Akopian T, Prichodko E, Kondratov I, Chukin M, Demina I, Galyamina M, Kamashev D, Vanyushkina A, Ladygina V, Levitskii S, Lazarev V, Govorun V (2012) Application of 
469

470

471

472

473

474

475

476

477

478

479

480

481

482

483

484

485

486

487

488

489

490

491

492

493

494

495

496

497

498

499

pathogenicity mechanisms in host-associated spiroplasmas. Journal of Proteome Research 11, 224-236.

Allen M, Ball B (1996) The incidence and world distribution of honey bee viruses. Bee World 77, 141-162.

Bankevich A, Nurk S, Antipov D, Gurevich AA, Dvorkin M, Kulikov AS, Lesin VM, Nikolenko SI, Pham S, Prjibelski AD, Pyshkin AV, Sirotkin AV, Vyahhi N, Tesler G, Alekseyev MA, Pevzner PA (2012) SPAdes: a new genome assembly algorithm and its applications to single-cell sequencing. Journal of Computational Biology 19, 455-477.

Blow F, Gioti A, Starns D, Ben-Yosef M, Pasternak Z, Jurkevitch E, Vontas J, Darby AC (2016) Draft genome sequence of the Bactrocera oleae symbiont "Candidatus Erwinia dacicola". Genome Announcements 4, e00896-16.

Bordenstein SR, Theis KR (2015) Host biology in light of the microbiome: ten principles of holobionts and hologenomes. PLoS Biology 13, e1002226.

Brettell L, Mordecai G, Schroeder D, Jones I, da Silva J, Vicente-Rubiano M, Martin S (2017) A comparison of deformed wing virus in deformed and asymptomatic honey bees. Insects $\mathbf{8}$, 28.

Browne HP, Forster SC, Anonye BO, Kumar N, Neville BA, Stares MD, Goulding D, Lawley TD (2016) Culturing of 'unculturable' human microbiota reveals novel taxa and extensive sporulation. Nature 533, 543-546.

Bruen TC, Philippe H, Bryant D (2006) A simple and robust statistical test for detecting the presence of recombination. Genetics 172, 2665-2681.

Calderone NW (2012) Insect pollinated crops, insect pollinators and US agriculture: trend analysis of aggregate data for the period 1992-2009. PLoS ONE 7, e37235.

Camacho C, Coulouris G, Avagyan V, Ma N, Papadopoulos J, Bealer K, Madden TL (2009) BLAST+: architecture and applications. BMC Bioinformatics 10, 421.

Cameron RC, Duncan EJ, Dearden PK (2013) Biased gene expression in early honeybee larval development. BMC Genomics 14, 903.

Caporaso JG, Lauber CL, Walters WA, Berg-Lyons D, Huntley J, Fierer N, Owens SM, Betley J, Fraser L, Bauer M, Gormley N, Gilbert JA, Smith G, Knight R (2012) Ultra-highthroughput microbial community analysis on the Illumina HiSeq and MiSeq platforms. The ISME Journal 6, 1621-1624. 
500 Chen Y, Higgins J, Feldlaufer M (2005) Quantitative real-time reverse transcription-PCR

501

502

503

504

505

506

507

508

509

510

511

512

513

514

515

516

517

518

519

520

521

522

523

524

525

526

527

528

529 analysis of deformed wing virus infection in the honeybee (Apis mellifera L). Applied and Environmental Microbiology 71, 436-441.

Clark T (1977) Spiroplasma sp, a new pathogen in honey bees. Journal of Invertebrate Pathology 113, 112-113.

Colbourne JK, Pfrender ME, Gilbert D, Thomas WK, Tucker A, Oakley TH, Tokishita S, Aerts A, Arnold GJ, Basu MK, Bauer DJ, Caceres CE, Carmel L, Casola C, Choi J-H, Detter JC, Dong Q, Dusheyko S, Eads BD, Frohlich T, Geiler-Samerotte KA, Gerlach D, Hatcher P, Jogdeo S, Krijgsveld J, Kriventseva EV, Kultz D, Laforsch C, Lindquist E, Lopez J, Manak JR, Muller J, Pangilinan J, Patwardhan RP, Pitluck S, Pritham EJ, Rechtsteiner A, Rho M, Rogozin IB, Sakarya O, Salamov A, Schaack S, Shapiro H, Shiga Y, Skalitzky C, Smith Z, Souvorov A, Sung W, Tang Z, Tsuchiya D, Tu H, Vos H, Wang M, Wolf YI, Yamagata H, Yamada T, Ye Y, Shaw JR, Andrews J, Crease TJ, Tang H, Lucas SM, Robertson HM, Bork P, Koonin EV, Zdobnov EM, Grigoriev IV, Lynch M, Boore JL (2011) The ecoresponsive genome of Daphnia pulex. Science 331, 555-561.

Conte YL, Ellis M, Ritter W (2010) Varroa mites and honey bee health: can Varroa explain part of the colony losses?. Apidologie 41, 353-363.

Cox-Foster DL, Conlan S, Holmes EC, Palacios G, Evans JD, Moran NA, Quan P-L, Briese T, Hornig M, Geiser DM, Martinson V, vanEngelsdorp D, Kalkstein AL, Drysdale A, Hui J, Zhai J, Cui L, Hutchison SK, Simons JF, Egholm M, Pettis JS, Lipkin WI (2007) A metagenomic survey of microbes in honey bee colony collapse disorder. Science 318, $283-$ 287.

Darling AE, Mau B, Perna NT (2010) Progressivemauve: multiple genome alignment with gene gain, loss and rearrangement. PLOS ONE 5, e11147.

Delcher AL, Bratke KA, Powers EC, Salzberg SL (2007) Identifying bacterial genes and endosymbiont DNA with Glimmer. Bioinformatics 23, 673-679.

Delwart EL (2007) Viral metagenomics. Reviews in Medical Virology 17, 115-131.

Denver DR, Brown AMV, Howe DK, Peetz AB, Zasada IA (2016) Genome skimming: a rapid approach to gaining diverse biological insights into multicellular pathogens. PLoS Pathogens 12, e1005713. 
530 Doublet V, Poeschl Y, Gogol-Döring A, Alaux C, Annoscia D, Aurori C, Barribeau SM,

531

532

533

534

535

536

537

538

539

540

541

542

543

544

545

546

547

548

549

550

551

552

553

554

555

556

557

558

559 Bedoya-Reina OC, Brown MJF, Bull JC, Flenniken ML, Galbraith DA, Genersch E, Gisder S, Grosse I, Holt HL, Hultmark D, Lattorff HMG, Conte YL, Manfredini F, McMahon DP, Moritz RFA, Nazzi F, Niño EL, Nowick K, van Rij RP, Paxton RJ, Grozinger CM (2017) Unity in defence: honeybee workers exhibit conserved molecular responses to diverse pathogens. BMC Genomics 18, 207.

Douglas AE (2014) Multiorganismal insects: diversity and function of resident microorganisms. Annual Review of Entomology 60, 1-18.

Douglas AE, Werren JH (2016) Holes in the hologenome: why host-microbe symbioses are not holobionts. mBio 7, e02099-15.

Duron O, Bouchon D, Boutin S, Bellamy L, Zhou L, Engelstädter J, Hurst GDD (2008) The diversity of reproductive parasites among arthropods: Wolbachia do not walk alone. BMC Biology 6, 27.

Emms DM, Kelly S (2015) OrthoFinder: solving fundamental biases in whole genome comparisons dramatically improves orthogroup inference accuracy. Genome Biology 16, 157.

Endo A, Futagawa-Endo Y, Dicks LM (2009) Isolation and characterization of fructophilic lactic acid bacteria from fructose-rich niches. Systematic and Applied Microbiology 32, 593-600.

Endo A, Salminen S (2013) Honeybees and beehives are rich sources for fructophilic lactic acid bacteria. Systematic and Applied Microbiology 36, 444-448.

Engel P, Kwong WK, McFrederick Q, Anderson KE, Barribeau SM, Chandler JA, Cornman RS, Dainat J, de Miranda JR, Doublet V, Emery O, Evans JD, Farinelli L, Flenniken ML, Granberg F, Grasis JA, Gauthier L, Hayer J, Koch H, Kocher S, Martinson VG, Moran N, Munoz-Torres M, Newton I, Paxton RJ, Powell E, Sadd BM, Schmid-Hempel P, SchmidHempel R, Song SJ, Schwarz RS, vanEngelsdorp D, Dainat B (2016) The bee microbiome: impact on bee health and model for evolution and ecology of host-microbe interactions. mBio 7, e02164-15.

Engel P, Martinson VG, Moran NA (2012) Functional diversity within the simple gut microbiota of the honey bee. Proceedings of the National Academy of Sciences of the United States of America 109, 11002-11007. 
560 Evans JD, Schwarz RS (2011) Bees brought to their knees: microbes affecting honey bee health.

561

562

563

564

565

566

567

568

569

570

571

572

573

574

575

576

577

578

579

580

581

582

583

584

585

586

587

588
Trends in Microbiology 19, 614-620.

Felis GE, Dellaglio F (2007) Taxonomy of lactobacilli and bifidobacteria. Current Issues in Intestinal Microbiology 8, 44.

Fierst JL, Murdock DA, Thanthiriwatte C, Willis JH, Phillips PC (2017) Metagenome-assembled draft genome sequence of a novel microbial Stenotrophomonas maltophilia strain isolated from Caenorhabditis remanei tissue. Genome Announcements 5, e01646-16.

Genersch E, Aubert M (2010) Emerging and re-emerging viruses of the honey bee (Apis mellifera L). Veterinary Research 41, 54.

Gerth M, Gansauge M-T, Weigert A, Bleidorn C (2014) Phylogenomic analyses uncover origin and spread of the Wolbachia pandemic. Nature Communications 5, 5117.

Goodwin S, McPherson JD, McCombie WR (2016) Coming of age: ten years of next-generation sequencing technologies. Nature Reviews Genetics 17, 333-351.

He Z, Zhang H, Gao S, Lercher MJ, Chen W-H, Hu S (2016) Evolview v2: an online visualization and management tool for customized and annotated phylogenetic trees. Nucleic Acids Research 44, W236-W241.

Kircher M, Kelso J (2010) High-throughput DNA sequencing - concepts and limitations. BioEssays 32, 524-536.

Klee J, Besana AM, Genersch E, Gisder S, Nanetti A, Tam DQ, Chinh TX, Puerta F, Ruz JM, Kryger P, Message D, Hatjina F, Korpela S, Fries I, Paxton RJ (2007) Widespread dispersal of the microsporidian Nosema ceranae, an emergent pathogen of the western honey bee, Apis mellifera. Journal of Invertebrate Pathology 96, 1-10.

Kumar S, Jones M, Koutsovoulos G, Clarke M, Blaxter ML (2013) Blobology: exploring raw genome data for contaminants, symbionts and parasites using taxon-annotated GCcoverage plots. Frontiers in Genetics 4, 237.

Kwong WK, Medina LA, Koch H, Sing K-W, Soh EJY, Ascher JS, Jaffé R, Moran NA (2017) Dynamic microbiome evolution in social bees. Science Advances 3, e1600513.

Kwong WK, Moran NA (2016) Gut microbial communities of social bees. Nature Reviews Microbiology 14, 374-384. 
589 Lanfear R, Calcott B, Ho SYW, Guindon S (2012) Partitionfinder: combined selection of 590 partitioning schemes and substitution models for phylogenetic analyses. Molecular Biology $591 \quad$ and Evolution 29, 1695-701.

592 Lanzi G, de Miranda JR, Boniotti MB, Cameron CE, Lavazza A, Capucci L, Camazine SM, 593 Rossi C (2006) Molecular and biological characterization of deformed wing virus of 594 honeybees (Apis mellifera L). Journal of Virology 80, 4998-5009.

595 Li D, Liu C-M, Luo R, Sadakane K, Lam T-W (2015) MEGAHIT: an ultra-fast single-node 596 solution for large and complex metagenomics assembly via succinct de Bruijn graph. Bioinformatics 31, 1674-1676.

Li H, Handsaker B, Wysoker A, Fennell T, Ruan J, Homer N, Marth G, Abecasis G, Durbin R, 1000 Genome Project Data Processing Subgroup (2009) The sequence alignment/map

601 format and SAMtools. Bioinformatics 25, 2078-2079.

Liang ZS, Nguyen T, Mattila HR, Rodriguez-Zas SL, Seeley TD, Robinson GE (2012) Molecular determinants of scouting behavior in honey bees. Science 335, 1225-1228.

Liu F, Li W, Li Z, Zhang S, Chen S, Su S (2011) High-abundance mRNAs in Apis mellifera: comparison between nurses and foragers. Journal of Insect Physiology 57, 274-279.

Lo W-S, Chen L-L, Chung W-C, Gasparich GE, Kuo C-H (2013) Comparative genome analysis of Spiroplasma melliferum IPMB4A, a honeybee-associated bacterium. BMC Genomics $14,22$.

Martin M (2011) Cutadapt removes adapter sequences from high-throughput sequencing reads. EMBnet.journal 17, 10-12.

Martin SJ, Highfield AC, Brettell L, Villalobos EM, Budge GE, Powell M, Nikaido S, Schroeder DC (2012) Global honey bee viral landscape altered by a parasitic mite. Science 336,

613 Martinson VG, Magoc T, Koch H, Salzberg SL, Moran NA (2014) Genomic features of a 614 bumble bee symbiont reflect its host environment. Applied and Environmental 615 Microbiology 80, 3793-3803.

616 McFall-Ngai M, Hadfield MG, Bosch TCG, Carey HV, Domazet-Lošo T, Douglas AE, Dubilier 617 N, Eberl G, Fukami T, Gilbert SF, Hentschel U, King N, Kjelleberg S, Knoll AH, Kremer 618 N, Mazmanian SK, Metcalf JL, Nealson K, Pierce NE, Rawls JF, Reid A, Ruby EG, 619 Rumpho M, Sanders JG, Tautz D, Wernegreen JJ (2013) Animals in a bacterial world, a 
620

621

622

623

624

625

626

627

628

629

630

631

632

633

634

635

636

637

638

639

640

641

642

643

644

645

646

647

648

649

650

new imperative for the life sciences. Proceedings of the National Academy of Sciences of the United States of America 110, 3229-3236.

McFrederick QS, Cannone JJ, Gutell RR, Kellner K, Plowes RM, Mueller UG (2013) Specificity between lactobacilli and hymenopteran hosts is the exception rather than the rule. Applied and Environmental Microbiology 79, 1803-12.

Merkling SH, Overheul GJ, van Mierlo JT, Arends D, Gilissen C, van Rij RP (2015) The heat shock response restricts virus infection in Drosophila. Scientific Reports 5, 12758.

Minh BQ, Nguyen MAT, von Haeseler A (2013) Ultrafast approximation for phylogenetic bootstrap. Molecular Biology and Evolution 30, 1188-1195.

Mokili JL, Rohwer F, Dutilh BE (2012) Metagenomics and future perspectives in virus discovery. Current Opinion in Virology 2, 63-77.

Moran NA, Sloan DB (2015) The Hologenome Concept: Helpful or Hollow?. PLOS Biology 13, e1002311.

Mordecai GJ, Wilfert L, Martin SJ, Jones IM, Schroeder DC (2016) Diversity in a honey bee pathogen: first report of a third master variant of the Deformed Wing Virus quasispecies. The ISME journal 10, 1264-1273.

Naeger NL, Robinson GE (2016) Transcriptomic analysis of instinctive and learned rewardrelated behaviors in honey bees. The Journal of Experimental Biology 219, 3554-3561.

Nawrocki EP (2009) Structural RNA homology search and alignment using covariance models. $\mathrm{PhD}$ Thesis, Washington University in Saint Louis.

Neumann P, Carreck NL (2010) Honey bee colony losses. Journal of Apicultural Research 49, $1-6$.

Nguyen L-T, Schmidt HA, von Haeseler A, Minh BQ (2015) IQ-TREE: a fast and effective stochastic algorithm for estimating maximum-likelihood phylogenies. Molecular Biology and Evolution 32, 268-274.

Nixon M (1982) Preliminary world maps of honeybee diseases and parasites. Bee World 63, 2342.

Oulas A, Pavloudi C, Polymenakou P, Pavlopoulos GA, Papanikolaou N, Kotoulas G, Arvanitidis C, Iliopoulos4 I (2015) Metagenomics: tools and insights for analyzing nextgeneration sequencing data derived from biodiversity studies. Bioinformatics and Biology Insights 9, 75 . 
651 Parks DH, Imelfort M, Skennerton CT, Hugenholtz P, Tyson GW (2015) CheckM: assessing the 652 quality of microbial genomes recovered from isolates, single cells, and metagenomes. 653 Genome Research 25, 1043-1055.

654 Peng Y, Leung HCM, Yiu SM, Chin FYL (2012) IDBA-UD: a de novo assembler for single-cell 655 656 1428.

Richardson MF, Weinert LA, Welch JJ, Linheiro RS, Magwire MM, Jiggins FM, Bergman CM (2012) Population genomics of the Wolbachia endosymbiont in Drosophila melanogaster. PLoS Genetics 8, e1003129.

Riesenfeld CS, Schloss PD, Handelsman J (2004) Metagenomics: genomic analysis of microbial communities. Annual Review of Genetics 38, 525-552.

Robinson MD, McCarthy DJ, Smyth GK (2009) edgeR: a Bioconductor package for differential expression analysis of digital gene expression data. Bioinformatics 26, 139-140.

Robinson MD, Smyth GK (2007) Small-sample estimation of negative binomial dispersion, with applications to SAGE data. Biostatistics 9, 321-332.

Salvetti E, Torriani S, Felis GE (2012) The genus Lactobacillus: a taxonomic update. Probiotics \& Antimicrobial Proteins 4, 217-226.

Salzberg SL, Hotopp J, Delcher A, Pop M, Smith D, Eisen M, Nelson W (2005) Serendipitous discovery of Wolbachia genomes in multiple Drosophila species. Genome Biology 6, R23.

Sboner A, Mu X, Greenbaum D, Auerbach RK, Gerstein MB (2011) The real cost of sequencing: higher than you think!. Genome Biology 12, 125.

Schloss PD, Westcott SL, Ryabin T, Hall JR, Hartmann M, Hollister EB, Lesniewski RA, Oakley BB, Parks DH, Robinson CJ, Sahl JW, Stres B, Thallinger GG, Horn DJV, Weber CF (2009) Introducing mothur: open-source, platform-independent, community-supported software for describing and comparing microbial communities. Applied and Environmental Microbiology 75, 7537-7541.

Schmieder R, Edwards R (2011) Fast identification and removal of sequence contamination from genomic and metagenomic datasets. PLOS ONE 6, e17288.

Schwarz RS, Bauchan GR, Murphy CA, Ravoet J, Graaf DC, Evans JD (2015) Characterization of two species of Trypanosomatidae from the honey bee Apis mellifera: Crithidia 
681

682

683

684

685

686

687

688

689

690

691

692

693

694

695

696

697

698

699

700

701

702

703

704

705

706

707

708

709

710

711

mellificae Langridge and McGhee, and Lotmaria passim n gen, n sp. Journal of Eukaryotic Microbiology 62, 567-583.

Sedlazeck FJ, Rescheneder P, von Haeseler A (2013) NextGenMap: fast and accurate read mapping in highly polymorphic genomes. Bioinformatics 29, 2790-2791.

Seemann T (2014) Prokka: rapid prokaryotic genome annotation. Bioinformatics 30, 2068-2069.

Siozios S, Cestaro A, Kaur R (2013) Draft genome sequence of the Wolbachia endosymbiont of Drosophila suzukii. Genome Announcements 1, e00032-13.

Sun Z, Harris HMB, McCann A, Guo C, Argimón S, Zhang W, Yang X, Jeffery IB, Cooney JC, Kagawa TF, Liu W, Song Y, Salvetti E, Wrobel A, Rasinkangas P, Parkhill J, Rea MC, O'Sullivan O, Ritari J, Douillard FP, Ross RP, Yang R, Briner AE, Felis GE, de Vos WM, Barrangou R, Klaenhammer TR, Caufield PW, Cui Y, Zhang H, O'Toole PW (2015) Expanding the biotechnology potential of lactobacilli through comparative genomics of 213 strains and associated genera. Nature Communications 6, 8322.

Tamarit D, Ellegaard KM, Wikander J, Olofsson T, Vasquez A, Andersson SGE (2015) Functionally structured genomes in Lactobacillus kunkeei colonizing the honey crop and food products of honeybees and stingless bees. Genome Biology and Evolution 7, 14551473.

R Core Team (2015) R: A language and environment for statistical computing.. R Foundation for Statistical Computing, Vienna, Austria.

VanEngelsdorp D, Evans JD, Saegerman C, Mullin C, Haubruge E, Nguyen BK, Frazier M, Frazier J, Cox-Foster D, Chen Y, Underwood R, Tarpy DR, Pettis JS (2009) Colony collapse disorder: a descriptive study. PLoS ONE 4, e6481.

Visser M, Bester R, Burger JT, Maree HJ (2016) Next-generation sequencing for virus detection: covering all the bases. Virology Journal 13, .

Whitfield CW (2003) Gene expression profiles in the brain predict behavior in individual honey bees. Science 302, 296-299.

Wilfert L, Long G, Leggett HC, Schmid-Hempel P, Butlin R, Martin SJM, Boots M (2016) Deformed wing virus is a recent global epidemic in honeybees driven by Varroa mites. Science 351, 594-597.

Wolfe KH, Li W-H (2003) Molecular evolution meets the genomics revolution. Nature Genetics 33, 255-265. 
712 Yue C (2005) RT-PCR analysis of Deformed wing virus in honeybees (Apis mellifera) and mites 713 (Varroa destructor). Journal of General Virology 86, 3419-3424.

714 Zioni N, Soroker V, Chejanovsky N (2011) Replication of Varroa destructor virus 1 (VDV-1)

715 and a Varroa destructor virus 1-deformed wing virus recombinant (VDV-1-DWV) in the 716 head of the honey bee. Virology 417, 106-112.

717 


\section{Figure legends}

719 Figure 1: Outline of screening procedure employed in this study. Each computational step is

720 listed in monospaced typeface as the leftmost column, details for each step are given on the right.

721 For the 'symbionts \& pathogens' database, microbial taxa are color-coded as follows: red-

722 pathogens, blue- gut symbionts, black- environmental/opportunistic symbionts. A second, more

723 detailed screen was performed for Lactobacillus symbionts only (marked with an asterisk, for

724 details see materials \& methods section). A detailed guide to replicate our analyses can be found

725 under https://github.com/gerthmicha/symbiont-sra.

726 Figure 2: Taxonomic annotation of contigs assembled from 'contaminated' Apis DNA short read

727 sequencing libraries. Bar chart shows the frequency of each taxonomic category assigned by best

728 BLAST matches against NCBI's 'nt' database, as the number of libraries in which that taxon was

729 detected among 993 DNA sequencing libraries. Bold categories are 'phyla', as defined in

730 https://www.ncbi.nlm.nih.gov/taxonomy, taxa in italics represent typical genera that were

731 recovered within each phylum. Please note that best blast hits to Arthropods were not included in

732 this plot. See Table S5 for a complete list.

733 Figure 3: 'Contamination' from Lactobacilli in Apis short read libraries. a) Maximum likelihood

734 tree of 72016 S rRNA sequences from Lactobacilli. Branch colors and the color of the outer

735 annotation circle correspond to Lactobacillus species groups according to Felis \& Dellaglio

736 (2007). Inner circle demarks taxa found Hymenoptera (grey squares) and in corbiculate apids

737 (honey bees and relatives, black squares). Lactobacillus sequences recovered in this study from

738 contaminated Apis libraries are labeled with blue triangles. The Lactobacilli typically associated

739 with honey bees (Firm-4, Firm-5, L. kunkeei) are further highlighted with a blue background

740 color. Two dotted blue lines denote the taxa of which whole draft genomes were recovered. See 
741 text for details. An interactive version of the tree containing all node labels is available under

742 http://www.evolgenius.info/evolview/\#shared/wZcKHbwJuT. b) Phylogeny of Lactobacillus

743 kunkeei strains based on maximum likelihood analyses of 947 concatenated single copy

744 orthologs (290,774 amino acid positions). Tree is rooted with Lactobacillus apinorum Fhon13

745 (taxon not shown). Strain names correspond to the names used in Tamarit et al. (2015; see Table

746 S3). Blue taxon label corresponds to the L. kunkeei strain recovered from 'contaminants' in

747 library SRR1046114. Bootstrap values are given on nodes. See Table S3 for sources of genomes.

748 c) Maximum likelihood tree of Fructobacillus (F.) and Leuconostoc (L.) species based on 435

749 concatenated single copy orthologs (145,069 amino acid positions). Tree is rooted with

750 Lactobacillus delbruecki. Numbers on nodes correspond to bootstrap values. Again, blue taxon

751 label denotes the Fructobacillus genome recovered from the 'contaminated' library

752 SRR1046114. Note that the phylogenetic distance between Fructobacillus fructosus and the

753 novel genome is similar to other between-species distances in this tree. See Table S3 for

754 accession numbers of all genomes used for phylogenetic analysis. d) Assembly statistics for the

755 two novel draft genomes recovered from library SRR1046114. Abbreviations: CDS- coding

756 sequences predicted with PROKKA, Comp. \& Cont.- completeness and contamination as

757 estimated with CheckM version 1.0.6 (Parks et al. 2015) based on the number of conserved

758 marker loci. Phylogenetic affiliations of the two strains are depicted in Fig. 3b and 3c,

759 respectively.

760 Figure 4: Characteristics of Spiroplasma melliferum isolated from a 'contaminated' Apis

761 mellifera sequencing library (SRR957082). a) Venn diagram illustrating the number of ortholog

762 groups shared between the novel strain and its closest sequenced relatives IBMB4A (Lo et al.

763 2013) and KC3 (Alexeev et al. 2012). b) Taxon-annotated GC-coverage plot of SRR951082 
764 metaassembly created with Blobology. Spiroplasma and Apis contigs can be differentiated by

765 coverage. c) Synteny across Spiroplasma melliferum genomes. Contigs from assemblies

766 SRR957082 and IPMB4A were ordered against KC3, the most complete of the three $S$.

767 melliferum genomes. Identical colors indicate syntenic blocks of the genomes. d) Phylogenetic

768 relationships within the genus Spiroplasma. Maximum likelihood tree is based on 206

769 concatenated loci (58,950 amino acid positions), numbers on branches correspond to bootstrap

770 values. Spiroplasma groups are highlighted with colors. The taxon label of the novel genome is

771 highlighted in bold. Accession numbers for all taxa are listed in Table S3.

772 Figure 5: Distribution of viral reads in Apis RNA sequencing libraries. a) Bar chart shows how

773 often each of the viruses associated with honey bees was detected with our approach. Results are

774 only shown for libraries with at least 500 reads of viral origin (236 in total). b) Bar chart shows

775 the distribution of relative proportions of viral reads for these 236 libraries.

776 Figure 6: Phylogeny of DWV sequences extracted from Apis RNA sequencing libraries. Tree is

777 based on 10,291 aligned positions and only sequences with at least 5,000bp were included.

778 Reference sequences and corresponding accession numbers are highlighted with bold typeface.

779 Colors at nodes indicate NCBI's BioProjects from which the sequences originated.

780 Figure 7: Example of differentially expressed honey bee genes in response to viral infection.

781 The relative expression levels of three genes that were determined to be significantly

782 differentially expressed with respect to viral infection status are plotted for one project. Each

783 library is represented by one dot, and lines correspond to $75 \%$ quartile, median, and $25 \%$ quartile

784 of all samples of a group, respectively. Expression levels for all investigated genes and projects

785 can be found in Figs. S2-S5 and Table S5. 


\section{Supplementary files}

787 Fig S1: Verification of screening approach employed here using the dataset of Engel et al.

788 (2012). All short reads from this dataset were mapped against Lactobacillus 16S reference

789 sequences as detailed in the materials \& methods section. Thus retrieved $16 \mathrm{~S}$ sequences are

790 highlighted with thick, dark blue lines. All other taxa in this tree are identical to the ones in Fig.

$7912 \mathrm{~A}$, as is the color scheme. Although the topology differs between these two Lactobacillus trees,

792 it is evident that the strains recovered from the Engel et al. (2012) dataset cluster within the

793 Firm-4 and Firm-5 Lactobacillus groups. Engel et al. (2012) essentially find the same (“These

794 distinct clusters reflect the eight dominant species with the two closely related Firmicutes (Firm-

7954 and Firm-5) [...]"; see also their Fig. 1c) using the programs MetaPhyler

796 (http://metaphyler.cbcb.umd.edu/) and IMG/M (https://img.jgi.doe.gov/) for taxonomic profiling.

797 Fig S2-S5: Gene expression of 20 honey bee genes loci across 4 investigated Apis RNA

798 sequencing projects (S2- PRJNA243651; S3- PRJNA292006; S4- PRJNA306498; S5-

799 PRJNA338450). Differentially expressed genes as determined with edgeR are marked with an

800 asterisk. See materials \& methods for more details.

801 Table S1: List of all DNA sequencing libraries investigated in this study, including associated

802 metadata.

803 Table S2: List of all RNA sequencing libraries investigated in this study, including associated 804 metadata.

805 Table S3: NCBI accession numbers for all genomes employed for comparative/phylogenetic 806 analyses of Lactobacillus kunkeei, Fructobacillus sp., and Spiroplasma sp. 
807 Table S4: Result of viral screening of RNA sequencing libraries. For 236 libraries with 500 or 808 more viral reads, absolute and relative numbers of each viral associate are provided, as well as 809 details on the project and tissues from which RNA was extracted (if available).

810 Table S5: Taxonomic summary of BLAST hits for contigs created in the screening of DNA 811 sequencing libraries. File created with a Blobtools script (see Materials \& methods).

812 Table S6: Complete result of analysis of differentially expressed genes in edgeR. See methods 813 for details.

814

815 


\section{Table 1 (on next page)}

Number of differentially expressed genes per investigated RNA sequencing project. 
1 Table 1: Number of differentially expressed genes per investigated RNA sequencing project.

\begin{tabular}{ccc} 
BioProject accession number & $\begin{array}{c}\text { Number of investigated RNA } \\
\text { sequencing libraries with/without } \\
\text { viruses }\end{array}$ & $\begin{array}{c}\text { Number of differentially } \\
\text { expressed genes* }\end{array}$ \\
\hline PRJNA243651 & $9 / 12$ & 7 \\
\hline PRJNA292006 & $3 / 2$ & 5 \\
\hline PRJNA306498 & $3 / 21$ & 5 \\
\hline PRJNA338450 & $17 / 19$ & 10 \\
\hline
\end{tabular}

2 * Out of 20 candidate loci investigated, see Table 2

3

4 


\section{Table 2 (on next page)}

Evidence for differential gene expression of honey bees in response to viral infections across four RNAseq projects (see Table 1).

20 candidate loci were investigated and differential expression was determined with edgeR (see text for details). 
1 Table 2: Evidence for differential gene expression of honey bees in response to viral infections 2 across four RNAseq projects (see Table 1). 20 candidate loci were investigated and differential 3 expression was determined with edgeR (see text for details).

\begin{tabular}{|c|c|c|}
\hline Locus & Description & $\begin{array}{l}\text { Number of projects in which } \\
\text { differentially expressed* }\end{array}$ \\
\hline LOC410087 & uncharacterized LOC410087 & 4 \\
\hline LOC408807 & uncharacterized LOC408807 & 3 \\
\hline LOC406114 & alpha-amylase & 2 \\
\hline LOC406142 & hymenoptaecin & 2 \\
\hline LOC724239 & $\begin{array}{l}\text { kynurenine/alpha-aminoadipate aminotransferase, } \\
\text { mitochondrial-like }\end{array}$ & 2 \\
\hline LOC724367 & protein lethal(2)essential for life-like & 2 \\
\hline LOC724654 & cytochrome b5 type B-like & 2 \\
\hline LOC726418 & flavin-containing monooxygenase FMO GS-OX-like 3-like & 2 \\
\hline $\mathrm{Vg}$ & vitellogenin & 2 \\
\hline Apid1 & apidaecin 1 & 1 \\
\hline Def2 & defensin 2 & 1 \\
\hline LOC406144 & abaecin & 1 \\
\hline LOC725017 & UDP-glycosyltransferase & 1 \\
\hline LOC725725 & uncharacterized LOC725725 & 1 \\
\hline Melt & melittin & 1 \\
\hline CYP6AQ1 & cytochrome P450 6AQ1 & 0 \\
\hline Def1 & defensin 1 & 0 \\
\hline LOC413908 & cytochrome P450 6AS12 & 0 \\
\hline LOC552832 & glycine N-methyltransferase-like & 0 \\
\hline LOC725158 & peptidoglycan recognition protein $\mathrm{S} 1$ & 0 \\
\hline
\end{tabular}




\section{Figure 1 (on next page)}

Outline of screening procedure employed in this study

Each computational step is listed in monospaced typeface as the leftmost column, details for each step are given on the right. For the 'symbionts $\&$ pathogens' database, microbial taxa are color-coded as follows: red-pathogens, blue- gut symbionts, blackenvironmental/opportunistic symbionts. A second, more detailed screen was performed for Lactobacillus symbionts only (marked with an asterisk, for details see materials $\&$ methods section). A detailed guide to replicate our analyses can be found under https://github.com/gerthmicha/symbiont-sra . 
Initial search QNCBI SRA

Separate by molecule

Exclude unwanted

Download via sra-tools or ftp server

Map against reference database

Extract \& assemble mapped reads

\section{"Apis"[Organism]}

DNA

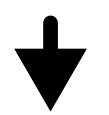

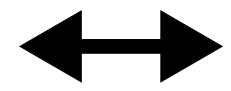

RNA

$\downarrow$ Amplicon sequencing

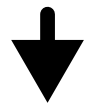

993 libraries from 17 Projects $\sim 24 \times 10^{9}$ reads

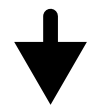

Metagenome sequencing

Viruses OR Varroa

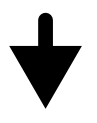

492 libraries from 58 Projects $\sim 23 \times 10^{9}$ reads

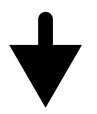

Symbionts \& pathogens rRNA genes Arsenophonus Melissococcus Ascosphaera Nosema

Bartonella Paenibacillus

Bifidobacterium Parasaccharibacter Crithidia Snodgrassella

Frischella Spiroplasma Gilliamella Wolbachia

Lactobacillus*

\section{Viruses}

Complete genomes

$\begin{array}{cc}\text { ABPV } & \text { KBV } \\ \text { BQCV } & \text { LSV } \\ \text { CBPV } & \text { SV } \\ \text { IAPV } & \text { SBPV } \\ \text { DWV type A,B,C }\end{array}$

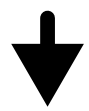

Downstream analyses (details in text) 
Figure 2 (on next page)

Taxonomic annotation of contigs assembled from 'contaminated' Apis DNA short read sequencing libraries

Bar chart shows the frequency of each taxonomic category assigned by best BLAST matches against NCBI's 'nt' database, as the number of libraries in which that taxon was detected among 993 DNA sequencing libraries. Bold categories are 'phyla', as defined in https://www.ncbi.nlm.nih.gov/taxonomy, taxa in italics represent typical genera that were recovered within each phylum. Please note that best blast hits to Arthropods were not included in this plot. See Table S5 for a complete list. 
Tenericutes

Spiroplasma

Microsporidia

Nosema

Streptophyta

Arabidopsis

Eukaryota-undef

Crithidia, Lotmaria

Bacteria-undef uncultured bacterium

Ascomycota Aspergillus, Penicillium

Actinobacteria

Bifidobacterium

Proteobacteria Bartonella, Frischella, Gilliamella, Snodgrassella

Firmicutes Lactobacillus 


\section{Figure 3 (on next page)}

'Contamination' from Lactobacilli in Apis short read libraries.

a) Maximum likelihood tree of $72016 \mathrm{~S}$ rRNA sequences from Lactobacilli. Branch colors and the color of the outer annotation circle correspond to Lactobacillus species groups according to Felis \& Dellaglio (2007). Inner circle demarks taxa found Hymenoptera (grey squares) and in corbiculate apids (honey bees and relatives, black squares). Lactobacillus sequences recovered in this study from contaminated Apis libraries are labeled with blue triangles. The Lactobacilli typically associated with honey bees (Firm-4, Firm-5, L. kunkeei) are further highlighted with a blue background color. Two dotted blue lines denote the taxa of which whole draft genomes were recovered. See text for details. An interactive version of the tree containing all node labels is available under

http://www.evolgenius.info/evolview/\#shared/wZcKHbw]uT. b) Phylogeny of Lactobacillus kunkeei strains based on maximum likelihood analyses of 947 concatenated single copy orthologs (290,774 amino acid positions). Tree is rooted with Lactobacillus apinorum Fhon13 (taxon not shown). Strain names correspond to the names used in Tamarit et al. (2015; see Table S3). Blue taxon label corresponds to the $L$. kunkeei strain recovered from 'contaminants' in library SRR1046114. Bootstrap values are given on nodes. See Table S3 for sources of genomes. c) Maximum likelihood tree of Fructobacillus (F.) and Leuconostoc (L.) species based on 435 concatenated single copy orthologs (145,069 amino acid positions). Tree is rooted with Lactobacillus delbruecki. Numbers on nodes correspond to bootstrap values. Again, blue taxon label denotes the Fructobacillus genome recovered from the 'contaminated' library SRR1046114. Note that the phylogenetic distance between Fructobacillus fructosus and the novel genome is similar to other between-species distances in this tree. See Table S3 for accession numbers of all genomes used for phylogenetic analysis. d) Assembly statistics for the two novel draft genomes recovered from library SRR1046114. Abbreviations: CDS- coding sequences predicted with PROKKA, Comp. \& Cont.-

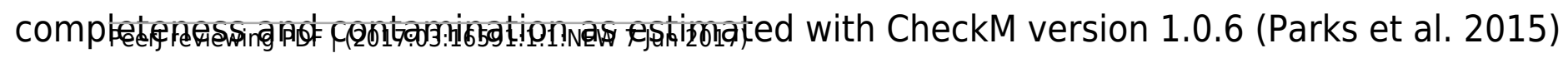


based on the number of conserved marker loci. Phylogenetic affiliations of the two strains are depicted in Fig. 3b and 3c, respectively. 
a) PeerJ
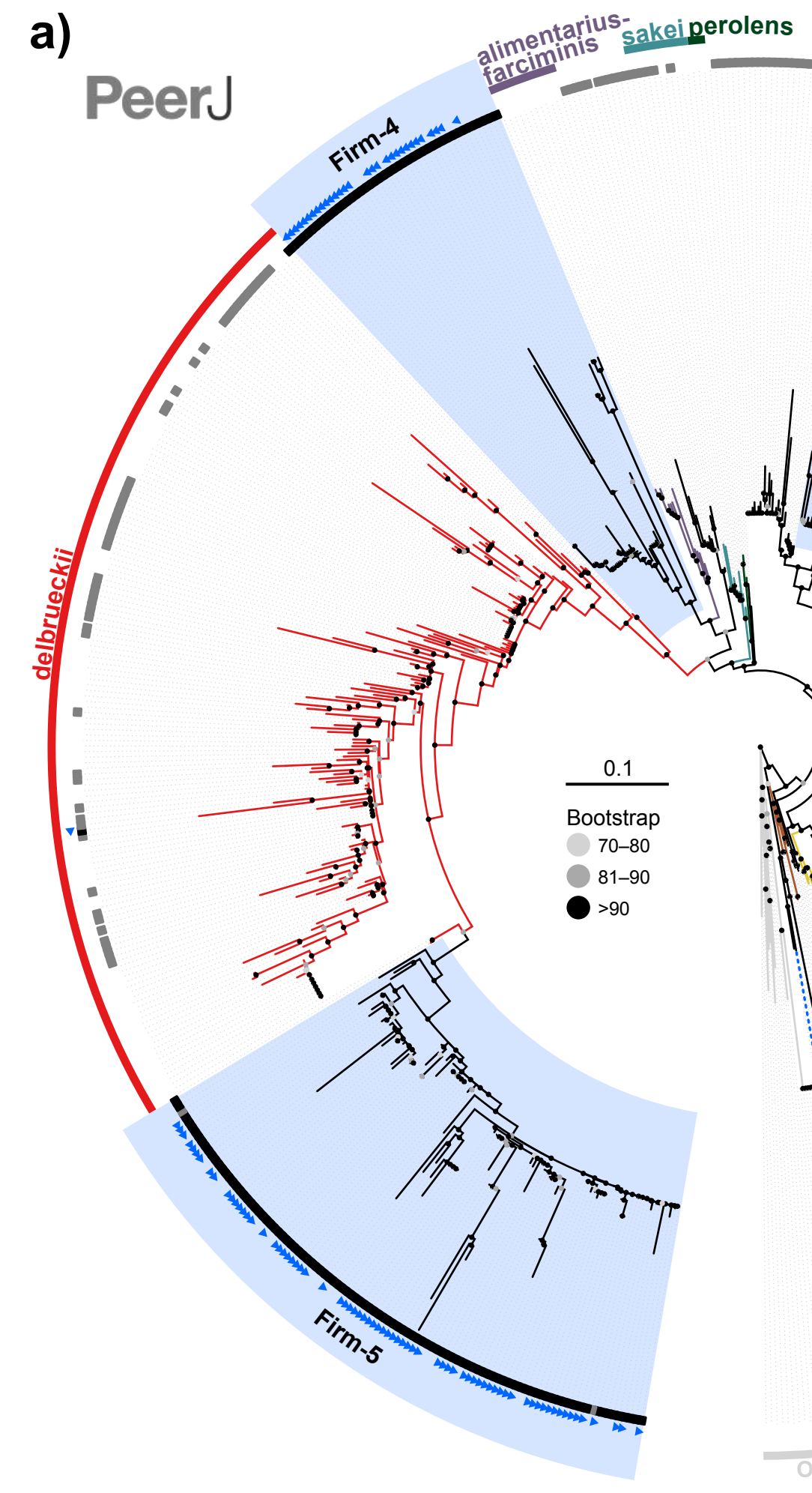

entariuskunkeei

Mintsuscript to be reviewed

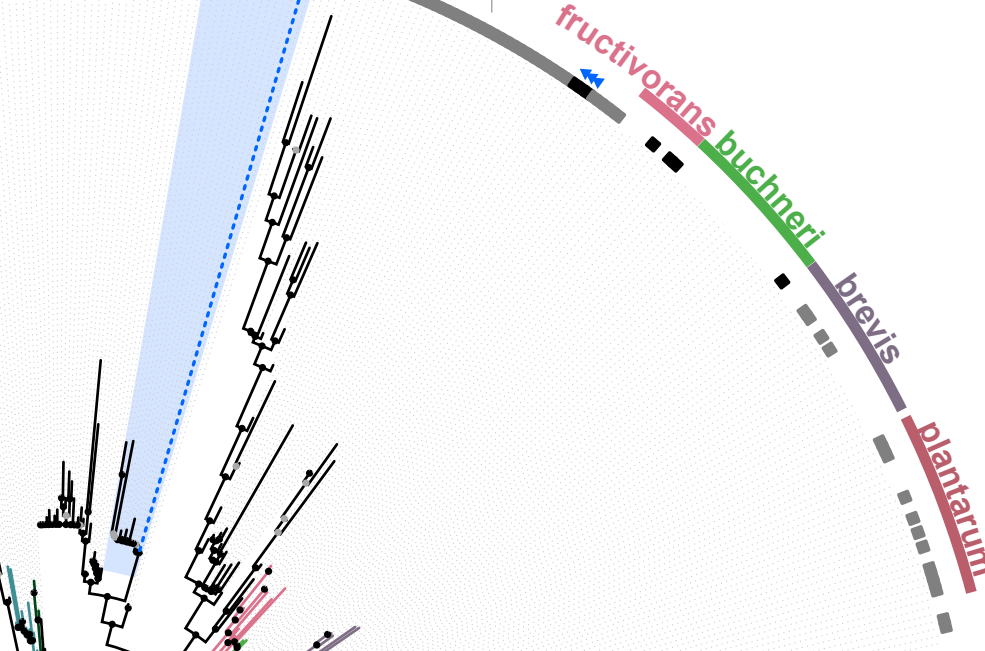

b)
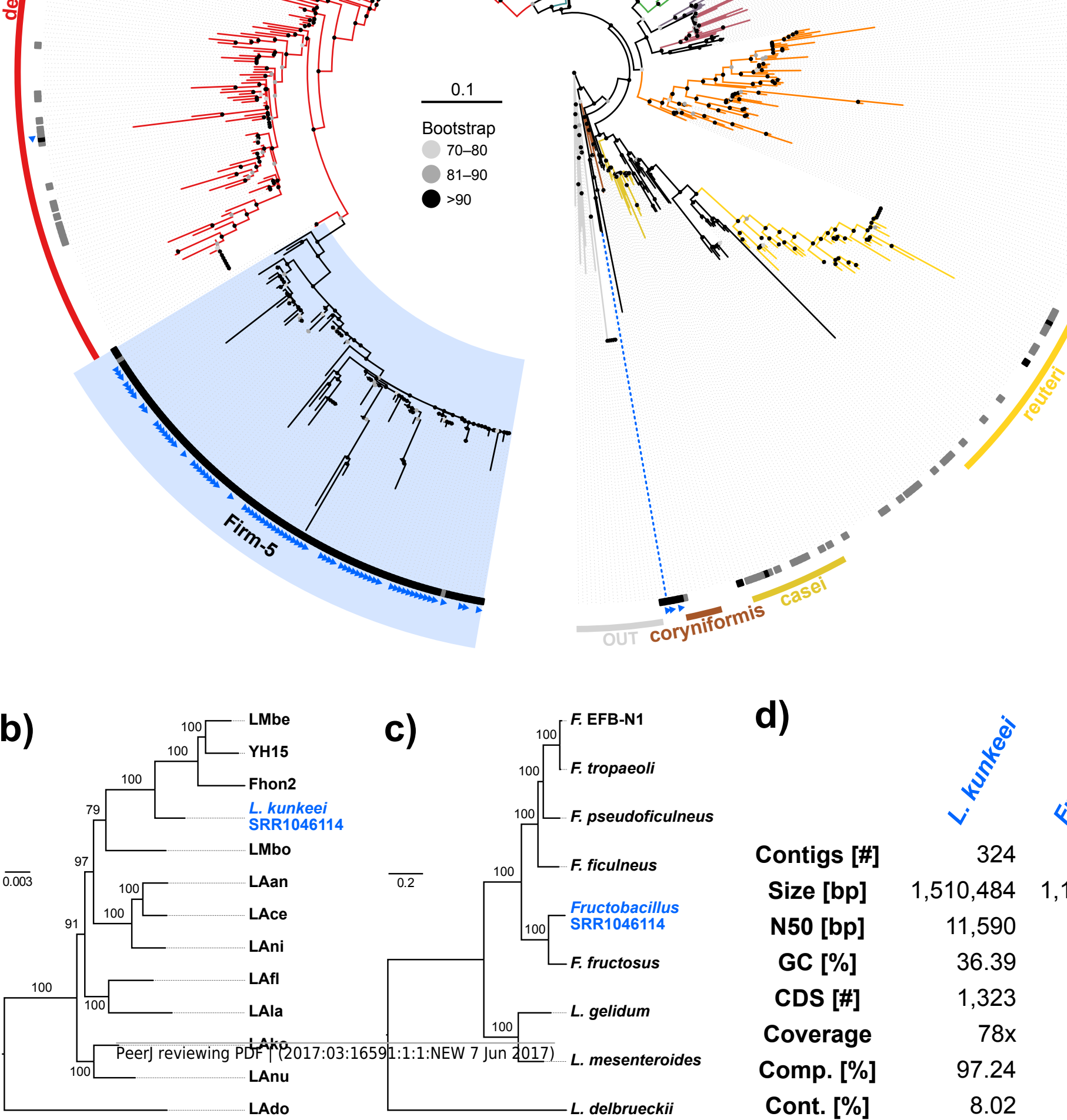

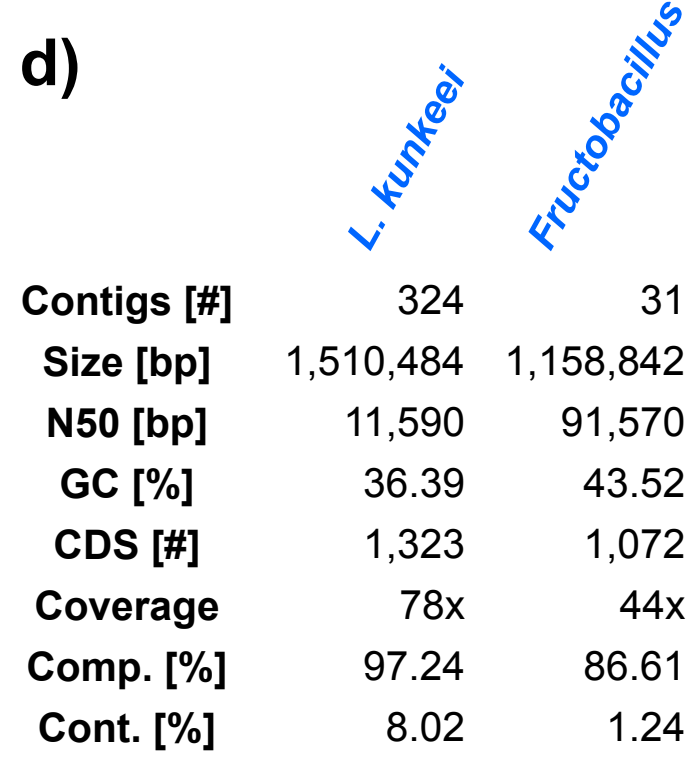




\section{Figure 4 (on next page)}

Characteristics of Spiroplasma melliferum isolated from a 'contaminated' Apis mellifera sequencing library (SRR957082)

a) Venn diagram illustrating the number of ortholog groups shared between the novel strain and its closest sequenced relatives IBMB4A (Lo et al. 2013) and KC3 (Alexeev et al. 2012). b) Taxon-annotated GC-coverage plot of SRR951082 metaassembly created with Blobology. Spiroplasma and Apis contigs can be differentiated by coverage. c) Synteny across Spiroplasma melliferum genomes. Contigs from assemblies SRR957082 and IPMB4A were ordered against $\mathrm{KC}$, the most complete of the three $S$. melliferum genomes. Identical colors indicate syntenic blocks of the genomes. d) Phylogenetic relationships within the genus Spiroplasma. Maximum likelihood tree is based on 206 concatenated loci $(58,950$ amino acid positions), numbers on branches correspond to bootstrap values. Spiroplasma groups are highlighted with colors. The taxon label of the novel genome is highlighted in bold. Accession numbers for all taxa are listed in Table S3. 
a)

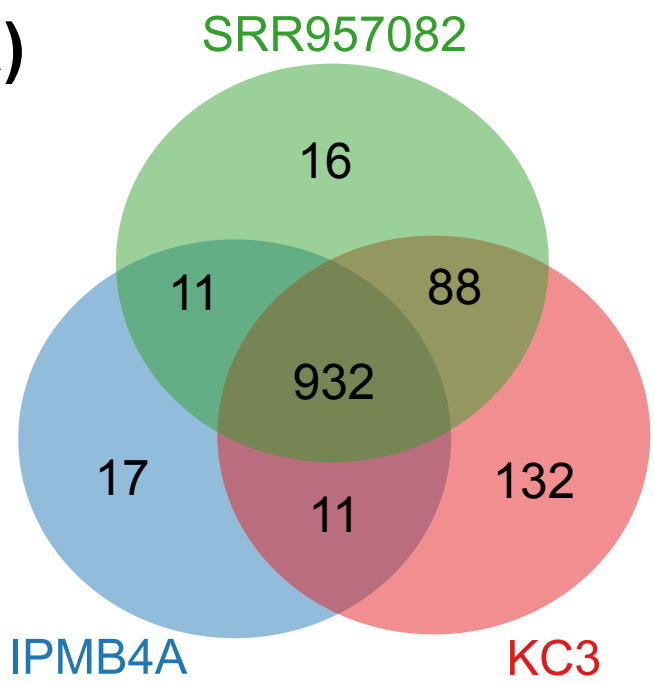

c)

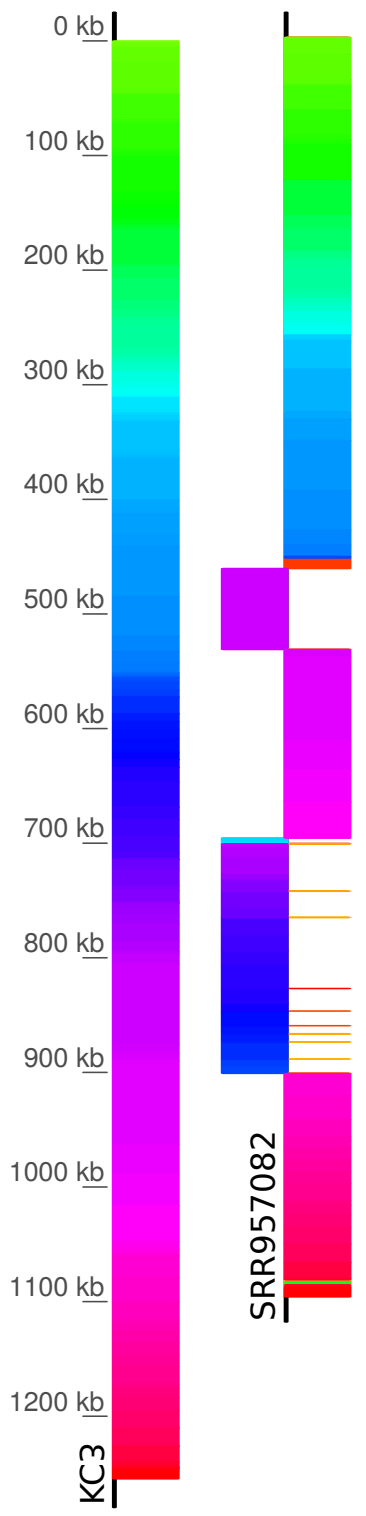

b)

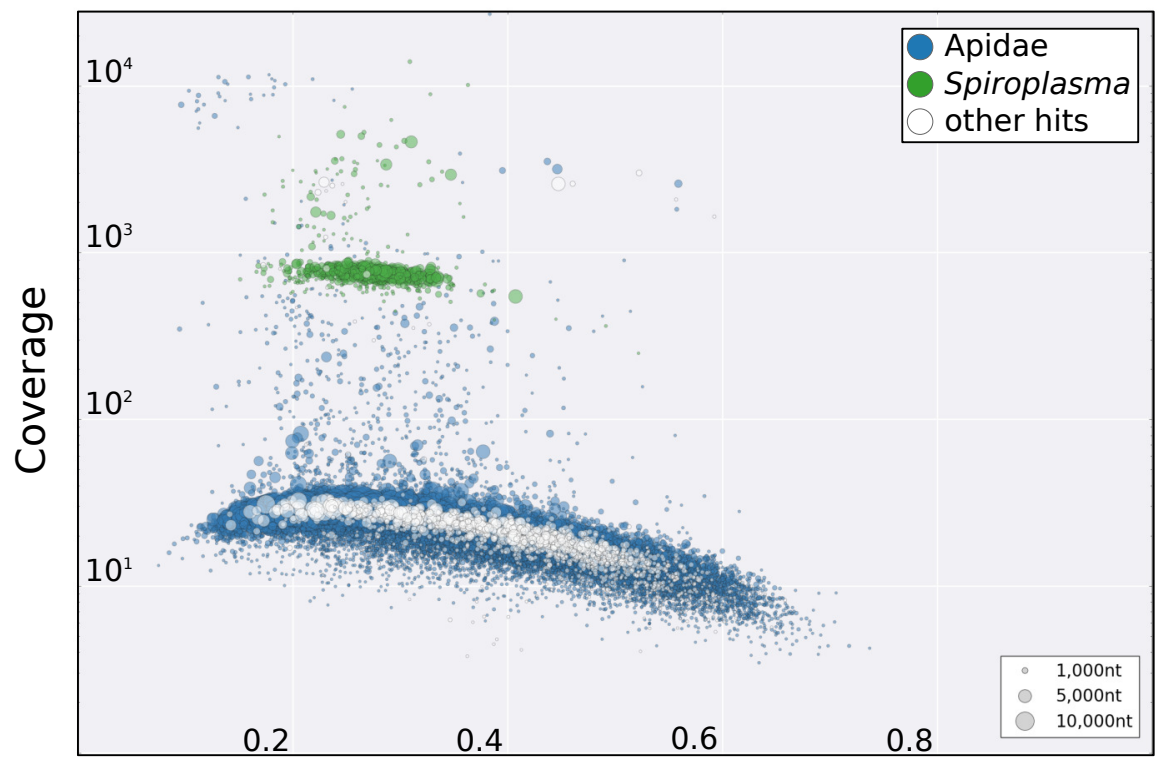

GC content

d)
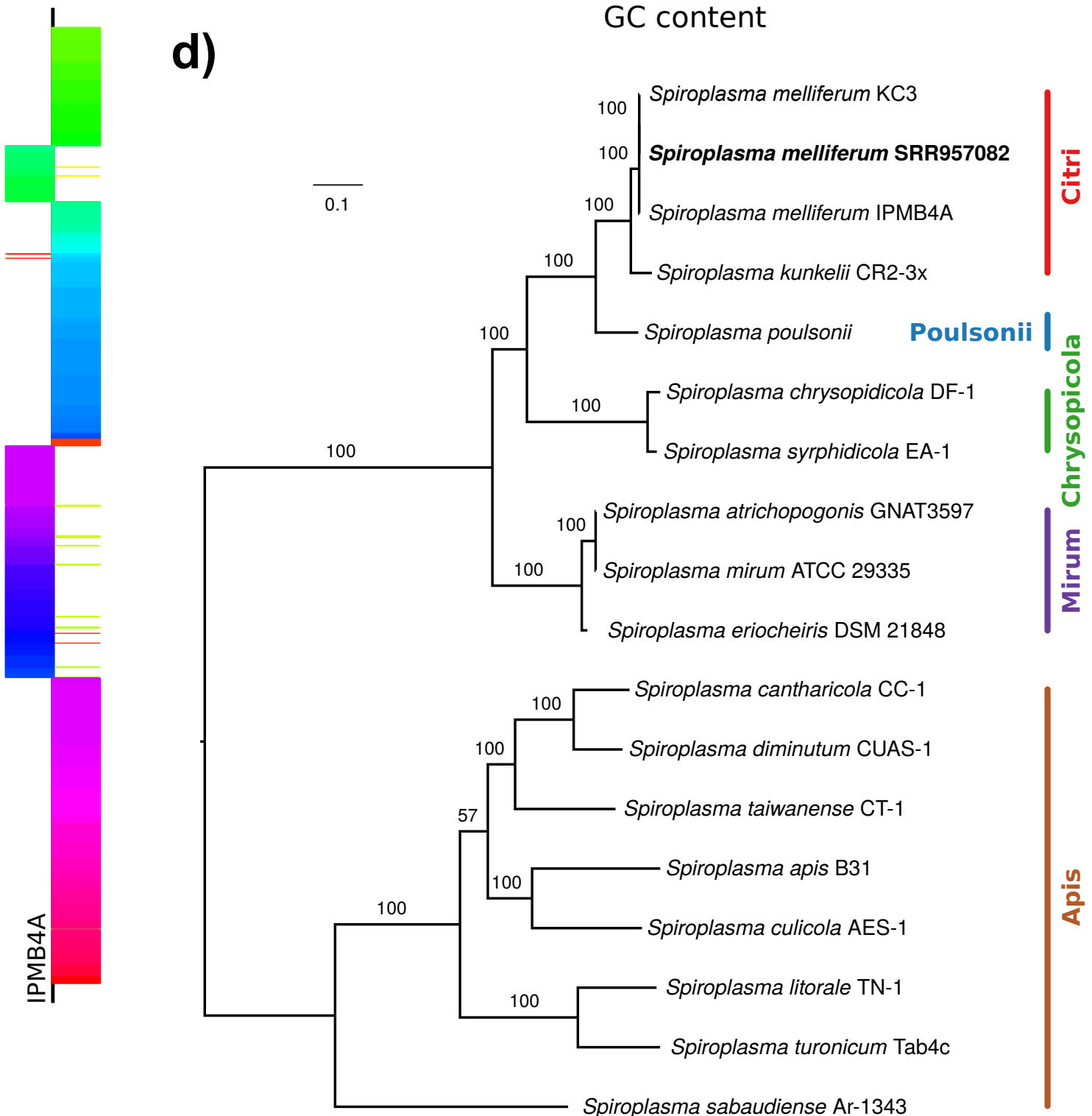
Figure $\mathbf{5}$ (on next page)

Distribution of viral reads in Apis RNA sequencing libraries.

a) Bar chart shows how often each of the viruses associated with honey bees was detected with our approach. Results are only shown for libraries with at least 500 reads of viral origin (236 in total). b) Bar chart shows the distribution of relative proportions of viral reads for these 236 libraries. 
a)

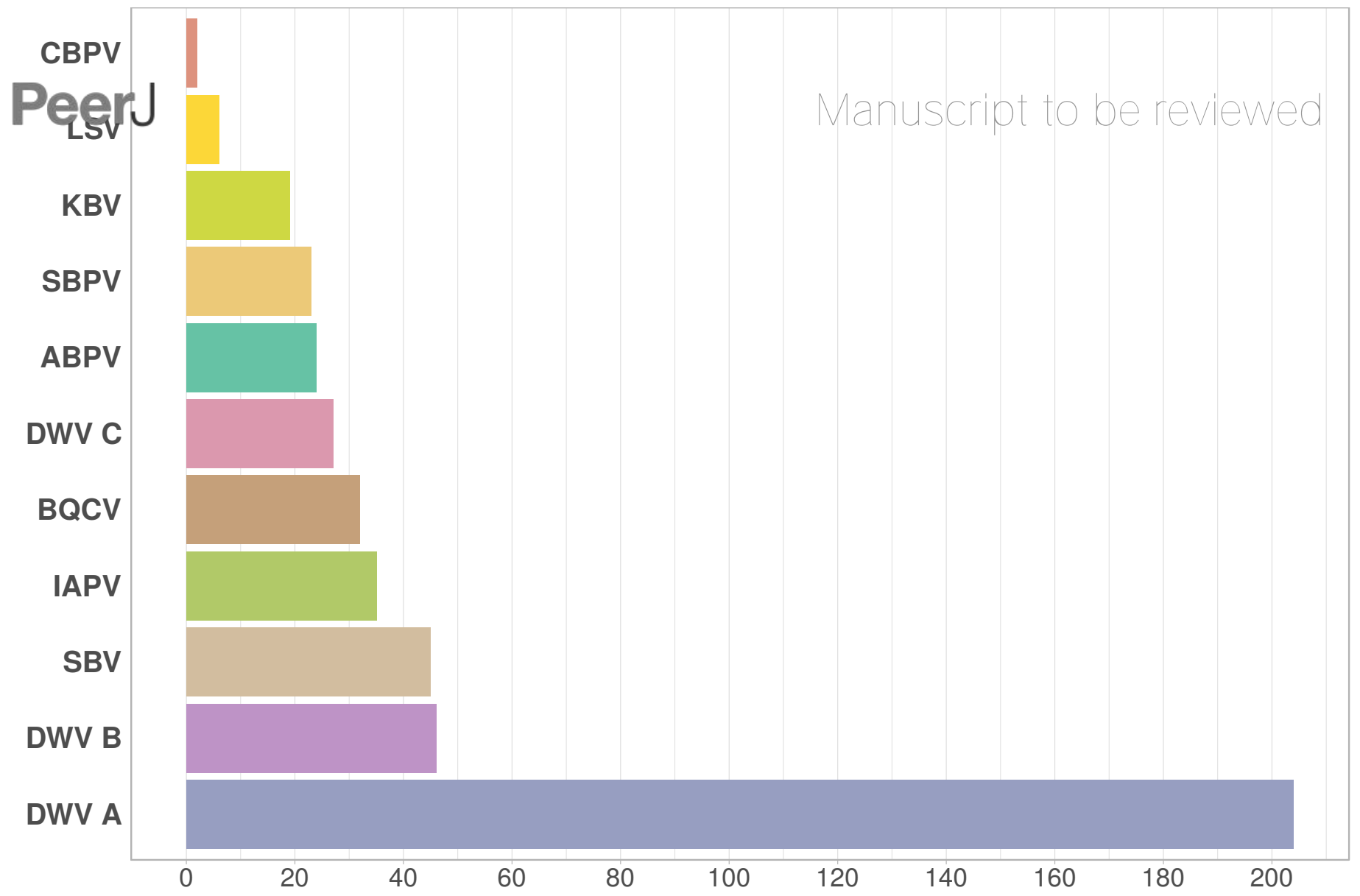

Number of RNA sequencing libraries with $\geq 500$ reads of specific viral associate

b)

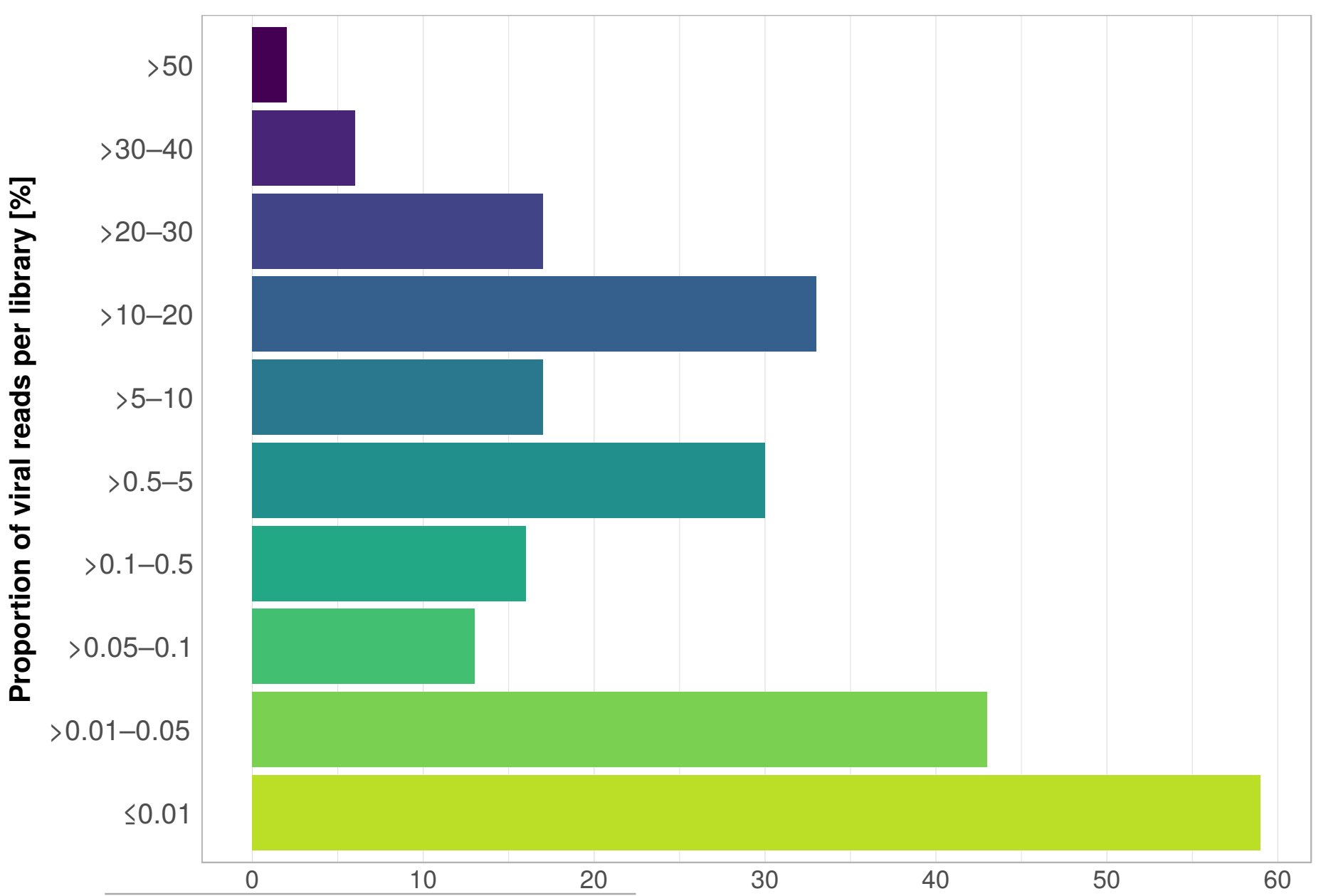




\section{Figure 6 (on next page)}

Phylogeny of DWV sequences extracted from Apis RNA sequencing libraries.

Tree is based on 10,291 aligned positions and only sequences with at least 5,000bp were included. Reference sequences and corresponding accession numbers are highlighted with bold typeface. Colors at nodes indicate NCBI's BioProjects from which the sequences originated. 
Degtstiap

70-80

81-90

$>90$

BioProject

PRJNA51481

PRJNA238833

PRJNA243651

PRJNA261549

PRJNA284414

PRJNA306498

PRJNA322424

PRJNA325930

PRJNA338450

SRX148699

PRJNA194157

PRJNA207482

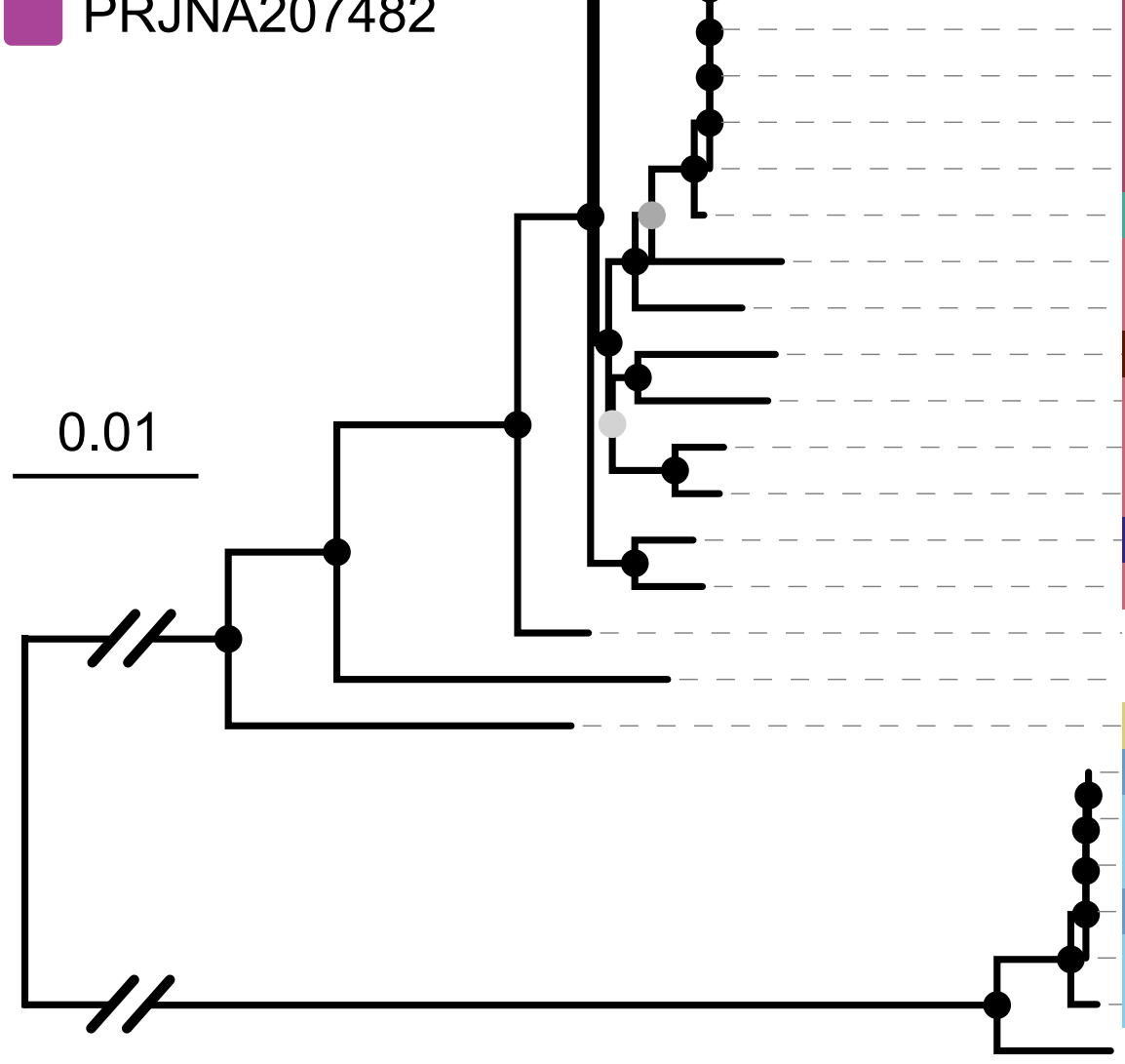

SRR893041

SRRR393042e reviemled

SRR893043

SRR893033

SRR893034

SRR893023

SRR893024

SRR893035

SRR893036

SRR893040

SRR893039

SRR893037

SRR893038

SRR1254947

SRR1254956

SRR1239303

SRR1239309

SRR1254957

SRR3033266

SRR3033254

SRR2034269

SRR1254946

SRR1254959-2

SRR1255009-2

SRR801788

SRR499919

SRR499992

SRR499882

SRR499920

SRR499993

SRR499994

SRR499995

SRR1582004

SRR4017785

SRR4017786

SRR3675070

SRR4017797

SRR4017792

SRR4017796

SRR063954

SRR4017752

NC04830 DWV A

NC05876 KV

SRR3569813

SRR1182312

SRR1239312

SRR1255009-1

SRR1182310

SRR1239311

SRR1254959-1

AY251269 DWV B 
Figure 7 (on next page)

Example of differentially expressed honey bee genes in response to viral infection.

The relative expression levels of three genes that were determined to be significantly differentially expressed with respect to viral infection status are plotted for one project. Each library is represented by one dot, and lines correspond to $75 \%$ quartile, median, and $25 \%$ quartile of all samples of a group, respectively. Expression levels for all investigated genes and projects can be found in Figs. S2-S5 and Table S5. 
